\title{
Option pricing and perfect hedging on correlated stocks
}

\author{
Jaume Masoliver团 and Josep Perelló \\ Departament de Física Fonamental, Universitat de Barcelona, \\ Diagonal, 647, 08028-Barcelona, Spain
}

\begin{abstract}
.
We develop a theory for option pricing with perfect hedging in an inefficient market model where the underlying price variations are autocorrelated over a time $\tau \geq 0$. This is accomplished by assuming that the underlying noise in the system is derived by an Ornstein-Uhlenbeck, rather than from a Wiener process. After obtaining an effective one-dimensional market model, we achieve a closed expression for the European call price within the Black-Scholes framework and find that our price is always lower than the Black-Scholes price. We obtain the same price if we start from a modified portfolio although now we get a different hedging strategy than that of Black-Scholes. We compare these strategies and study the sensitivity of the call price to several parameters where the correlation effects are also observed.
\end{abstract}

\section{Introduction}

Fischer Black and Myron Scholes (1973) and Robert Merton (1973a) obtained a fair option price assuming severe and strict theoretical conditions for the market behavior. The requirements under which these were developed include: (i) Absence of arbitrage opportunities, i.e., identical cashflows have identical values (Sharpe (1964); Cox and Ross (1976)). (ii) Efficient market hypothesis, i.e., the market incorporates instantaneously any information concerning future market evolution (Fama (1965)). (iii) Existence of a unique riskless strategy for a portfolio in a complete market (Markowitz (1952)). Due to the random character of stock market prices (Cootner (1964)), the implementation of these conditions, especially condition (ii), indicates that speculative prices are driven by white (i.e., delta-correlated) random processes. At this point, one has to choose between a Gaussian white process (Black and Scholes (1973); Merton (1973a)) or a white jump process. In this latter case and due to requirement (iii), the jump lengths also have to be known and fixed (Merton (1976)). There are no other choices for modelling market evolution if the above requirements and ideal conditions are to be obeyed (Cox and Ross (1976)).

From these three assumptions, condition (ii) is perhaps the most restrictive and, in fact, disagrees with empirical evidence since real markets are not efficient, at least at

$\ddagger$ Corresponding author. E-mail: jaume@ffn.ub.es 
short times (Grossman and Stiglitz (1980); Fama (1991)). Indeed, market efficiency is closely related to the assumption of totally uncorrelated price variations (white noise). But white noise is only an idealization since, in practice, no actual random process is completely white. For this reason, white processes are convenient mathematical objects valid only when the observation time is much larger than the autocorrelation time of the process[s]. And, analogously, the efficient market hypothesis is again a convenient assumption when the observation time is much larger than time spans in which "inefficiencies" (i.e., correlations, delays, etc....) occur.

Alternative models for describing empirical results of the market evolution have been suggested (Mandelbrot (1963); Fama (1963)). In each of these, an option price can be obtained only by relaxing some or even all of the initial Black-Scholes (B-S) assumptions (Figlewski (1989); Aurell et al (2000)). Our main purpose in this paper is to derive a nontrivial option price by relaxing the efficient market hypothesis and allowing for a finite, non-zero, correlation time of the underlying noise process. As a model for the evolution of the market we choose the Ornstein-Uhlenbeck $(\mathrm{O}-\mathrm{U})$ process (Uhlenbeck and Ornstein (1930)) for three reasons: (a) O-U noise is still a Gaussian random process with an arbitrary correlation time $\tau$ and it has the property that when $\tau=0$ the process becomes Gaussian white noise, as in the original Black-Scholes option case. (b) The O-U process is, by virtue of Doob's theorem, the only Gaussian random process which is simultaneously Markovian and stationary (Doob (1942)). In this sense the O-U process is the simplest generalization of Gaussian white-noise. (c) As we will see later on, the variance of random processes driven by $\mathrm{O}-\mathrm{U}$ noise seems to agree with the evolution of market variance, at least in some particular but relevant cases.

The Ornstein-Uhlenbeck process is not a newcomer in mathematical finance. For instance, it has already been proposed as a model for stochastic volatility case here is rather different since, contrary to SV models, we only have one source of noise. We therefore suggest the $\mathrm{O}-\mathrm{U}$ process as the driving noise for the underlying price dynamics when the volatility is still a deterministic quantity (Dumas et al (1998)).

The autocorrelation in the underlying driving noise is closely related to the predictability of asset returns, of which there seems to be ample evidence (Breen and Jagannathan (1989); Campbell and Hamao (1992)). Indeed, if for some particular stock the price variations are correlated during some time $\tau$, then the price at time $t_{2}$ will be related to the price at a previous time $t_{1}$ as long as the time span $t_{2}-t_{1}$ is not too long compared to the correlation time $\tau$. Hence correlation implies partial predictability. Other approaches to option pricing with predictable asset returns are based under the assumption the market is still driven by white noise and predictability is induced by the drift (Lo and Wang (1995)). Since the B-S formula is independent of the drift, these approaches apply B-S theory with a conveniently modified volatility. Our approach here is rather different because we assume the asset price variations driven by correlated noise

$\S$ Throughout this paper we will use the terms "correlation" and "autocorrelation" without distinction. II Hull and White (1987); Scott (1987); Stein and Stein (1991); Heston (1993); Ghysels et al (1996); Heston and Nandi (2000). 
-which implies some degree of predictability.

Summarizing, our purpose is to study option pricing and hedging in a more realistic framework that of white noise process presented by Black and Scholes. Our model includes colored noise and the dependence of the volatility on time. Both are empirically observed in real markets (Bouchaud and Potters (2000)). Empirical characteristic time scales are at least of the order of minutes and can affect option prices particularly when the exercising date is near and speculative fluctuations are more important. Presumably, this effect is negligible when correlation times are shorter (much shorter than time to expiration). In any case, it is interesting to know how, and by how much, the option price and its properties are modified when correlations in the underlying noise are significant.

The shortest way of getting the call price, and hence quantifying the effect of correlations on prices is by martingale methods. Unfortunately, this procedure does not guarantee that we obtain the fairest price since arbitrage and hedging are not included in this approach. It is therefore our main objective to generalize B-S theory not only to get a new call price but, more importantly, to obtain a hedging strategy that avoids risk and arbitrage opportunities.

From a technical point of view, we apply the B-S option pricing method after projecting the two-dimensional $\mathrm{O}-\mathrm{U}$ process onto a one-dimensional diffusion process with time varying volatility. As we will show, this projection allows us to maintain the conditions of a perfect hedging and the absence of arbitrage. Moreover, the price obtained using this way completely agrees with the price obtained using two alternative and different methods. One of them is based on martingale theory, and the other one develops a new option pricing with a modified portfolio containing secondary options instead of the underlying stock.

The paper is divided into eight sections. In Section 2 we present our twodimensional stochastic model for the underlying asset. In Section 3 we find the O-U projection onto the stock price correlated process. Section 1 concentrates on the B$\mathrm{S}$ option price derivation with the projected process, and Sections 5 and 6 show the consistency of this derivation by using two alternative methods for obtaining the option price. The greeks and the new hedging are presented in Section 0. Conclusions are drawn in Section 8 and technical details are left to the appendices.

\section{The asset model}

The standard assumption in option pricing theory is to assume that the underlying price $S(t)$ can be modelled as a one-dimensional diffusion process:

$$
\frac{d S(t)}{S(t)}=\mu d t+\sigma d W(t),
$$

where $W(t)$ is the Wiener process. In the original B-S theory both drift $\mu$ and volatility $\sigma$ are constants. Other models take $\mu=\mu(t, S)$ and $\sigma=\sigma(t, S)$ as functions of time and underlying price (Cox and Ross (1976); Bergman et al (1987)). The parameter $\sigma$ is assumed to be a random quantity in the SV models. 
Notice that if the time evolution of the underlying price is governed by Eq. (11) then $S(t)$ is an uncorrelated random process in the sense that its zero-mean return rate defined by $Z(t)=d \ln S / d t-\mu$ is driven by white noise, i.e., $E\left[Z\left(t_{1}\right) Z\left(t_{2}\right)\right]=\sigma^{2} \delta\left(t_{1}-t_{2}\right)$ where $\delta(t)$ is the Dirac delta function $\rceil$. Hence, the asset model immediately incorporates price return effects and meets the efficient market hypothesis.

As a first step, we assume that the underlying price is not driven by the Wiener process $W(t)$ but by O-U noise $V(t)$. In other words, we say that $S(t)$ obeys a singular two-dimensional diffusion

$$
\begin{aligned}
& \frac{d S(t)}{S(t)}=\mu d t+V(t) d t \\
& d V(t)=-\frac{V(t)}{\tau} d t+\frac{\sigma}{\tau} d W(t),
\end{aligned}
$$

where $\tau \geq 0$ is the correlation time. More precisely, $V(t)$ is $\mathrm{O}-\mathrm{U}$ noise in the stationary regime, which is a Gaussian colored noise with zero mean and correlation function:

$$
E\left[V\left(t_{1}\right) V\left(t_{2}\right)\right]=\frac{\sigma^{2}}{2 \tau} e^{-\left|t_{1}-t_{2}\right| / \tau} .
$$

We call the process defined by Eqs. (2)-(3) singular diffusion because, contrary to SV models, the Wiener driving noise $W(t)$ only appears in one of the equations, and this results in a singular diffusion matrix (Gardiner (1985)). Observe that we now deal with autocorrelated stock prices since the zero-mean return rate $Z(t)$ is colored noise, i.e., $E\left[Z\left(t_{1}\right) Z\left(t_{2}\right)\right]=\left(\sigma^{2} / 2 \tau\right) \exp \left[-\left|t_{1}-t_{2}\right| / \tau\right]$. Note that when $\tau=0$ this correlation goes to $\sigma^{2} \delta\left(t_{1}-t_{2}\right)$ and we thus recover the one-dimensional diffusion discussed above. Therefore the case of positive $\tau$ is a measure of the inefficiencies of the market.

There is an alternative, and sometimes more convenient, way of writing the above equations using the asset return $R(t)$ defined by

$$
R(t)=\ln \left[S(t) / S_{0}\right],
$$

where $S_{0}=S\left(t_{0}\right)$ and $t_{0}$ is the time at which we start observing the process (2)-(3). Without loss of generality this time can be set equal to zero (see Appendix A). Instead of Eqs. (2)-(3), we may have

$$
\begin{aligned}
& \frac{d R(t)}{d t}=\mu+V(t) \\
& \frac{d V(t)}{d t}=\frac{1}{\tau}[-V(t)+\sigma \xi(t)],
\end{aligned}
$$

where $\xi(t)=d W(t) / d t$ is Gaussian white noise defined as the derivative of the Wiener process. This process exists in the sense of generalized random functions (Lighthill (1958); Stratonovich (1963)). The combination of relations in Eqs. (5) and (6) leads to a second-order stochastic differential equation for $R(t)$

$$
\tau \frac{d^{2} R(t)}{d t^{2}}+\frac{d R(t)}{d t}=\mu+\sigma \xi(t)
$$

I We recall that $\delta(x)$ is a generalized function with the properties: $\delta(x)=0$ for $x \neq 0$ and $\int_{-\infty}^{\infty} \delta(x) d x=1$ (Lighthill (1958); Stratonovich (1963)). 
From this equation, we clearly see that when $\tau=0$ we recover the one-dimensional diffusion case (1) $)$. We also observe that the O-U process $V(t)$ is the random part of the return velocity, $d R / d t$, and we will often refer to $V(t)$ as the "velocity" of the return process $R(t)$.

In Appendix A, we give explicit expressions for $V(t)$ and for the return $R(t)$. We prove there that $R(t)$ is a non-stationary process with the conditional mean value

$$
m\left(t, V_{0}\right) \equiv E\left[R(t) \mid V_{0}\right]=\mu t+\tau\left(1-e^{-t / \tau}\right) V_{0},
$$

where $V(0) \equiv V_{0}$ is the initial velocity. The conditional return variance,

$$
K_{11}(t) \equiv E\left[\left(R(t)-m\left(t, V_{0}\right)\right)^{2} \mid V_{0}\right]
$$

is given by

$$
K_{11}(t)=\sigma^{2}\left[t-2 \tau\left(1-e^{-t / \tau}\right)+\frac{\tau}{2}\left(1-e^{-2 t / \tau}\right)\right] .
$$

We also give in Appendix A explicit expressions for the joint probability density function (pdf) $p(R, V, t)$, the marginal pdf's $p(R, t)$ and $p(V, t)$ of the second-order process $R(t)$, and the marginal pdf $p\left(S, t \mid S_{0}, t_{0}\right)$ of the underlying price $S(t)$. We also show that the velocity $V(t)$ is, in the stationary regime, distributed according to the normal density:

$$
p_{s t}(V)=\frac{1}{\sqrt{\pi \sigma^{2} / \tau}} e^{-\tau V^{2} / \sigma^{2}} .
$$

Suppose now that the initial velocitiy $V_{0}$ is random with mean value $E\left[V_{0}\right]$ and variance $\operatorname{Var}\left[V_{0}\right]$. Thus, the return unconditional mean and variance read

$$
\begin{array}{ll}
E[R(t)] & =\mu t+\tau\left(1-e^{-t / \tau}\right) E\left[V_{0}\right], \\
E\left[\left(R(t)-E(R(t))^{2}\right]\right. & =K_{11}(t)+\tau\left(1-e^{-t / \tau}\right) \operatorname{Var}\left[V_{0}\right]
\end{array}
$$

If, in addition, we assume that the initial velocity $V_{0}$ is in the stationary regime then $E\left[V_{0}\right]=0$ and $\operatorname{Var}\left[V_{0}\right]=\sigma^{2} / 2 \tau$. In this case, the return unconditional mean value is

$$
m(t) \equiv E[R(t)]=\mu t
$$

and the return unconditional variance

$$
\kappa(t) \equiv E\left[(R(t)-m(t))^{2}\right]
$$

reads (cf. Eq. (9))

$$
\kappa(t)=\sigma^{2}\left[t-\tau\left(1-e^{-t / \tau}\right)\right] .
$$

A consequence of Eq. (11) is that, when $t \ll \tau$, the variance behaves as

$$
\kappa(t) \sim\left(\sigma^{2} / 2 \tau\right) t^{2}, \quad(t \ll \tau) .
$$

Equation (11) also shows a crossover to ordinary diffusion (B-S case) when $t \gg \tau$ :

$$
\kappa(t) \sim \sigma^{2} t, \quad(t \gg \tau) .
$$

+ In the opposite case when $\tau=\infty$, Eq. (3) shows that $d V(t)=0$. Thus $V(t)$ is a constant, which we may equal to zero, and from Eq. (2) we have $S(t)=S_{0} e^{\mu t}$. Therefore, the underlying price evolves as a riskless security. Later on we will recover this deterministic case (see, for instance, Eq. (35)). 


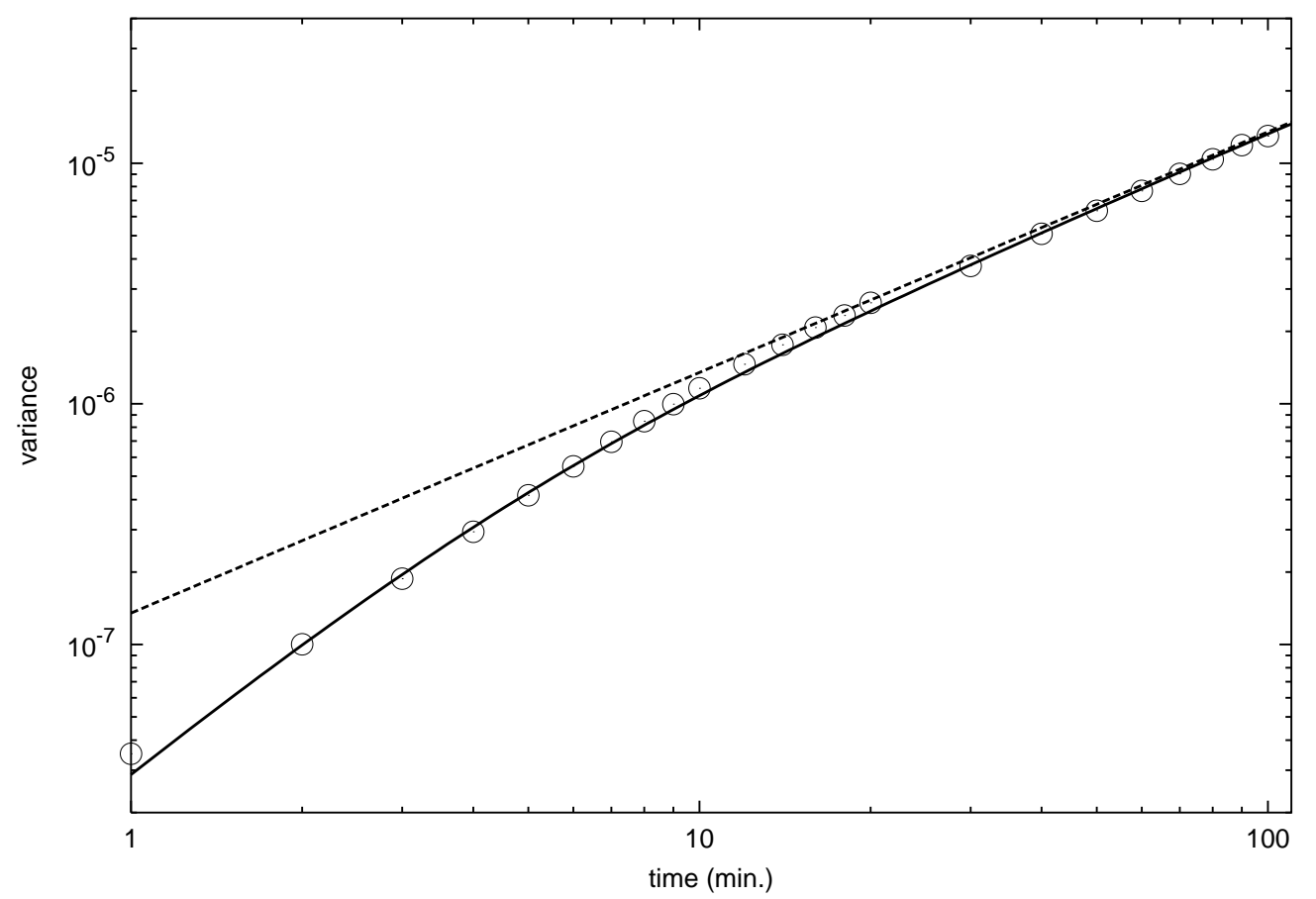

Figure 1. The variance of the underlying asset as a function of time (in logarithmic scale). Circles correspond to empirical variance of S\&P 500 cash index from 1988 to 1996. Solid line represents the theoretical variance, Eq. (7) with $\tau=2$ minutes. The dashed line is the B-S variance $\sigma^{2} t$. In both cases $\sigma=3.69 \times 10^{-4} \mathrm{~min}^{-1 / 2}$ which approximately corresponds to an annual volatility $\sigma=11 \%$.

In Fig. 1, we plot $\kappa(t)$ along with the empirical variance from data of the S\&P 500 cash index during the period January 1988-December 1996 The dashed line represents results obtained by assuming normal-diffusion $\kappa(t) \propto t$. Observe that the empirical variance is very well fitted by our theoretical variance $\kappa(t)$ for a correlation time $\tau=2$ minutes. Furthermore, the result of this correlation affects the empirical volatility for around 100 minutes. These times are probably too small to affect call price to any quantifiable extent. However, the S\&P 500 is one of the most liquid, and therefore most efficient, markets. Consequently, the effect of correlations in any other less efficient market might significantly influence option prices and hedging strategies, and this is the main motivation for this work.

\section{The projected process}

One may argue that the $\mathrm{O}-\mathrm{U}$ process (2)-(3) is an inadequate asset model since the share price $S(t)$ given by Eq. (2) is a continuous random process with bounded variations. As Harrison et al (1984) showed, continuous processes with bounded variations allow

* Tick by tick data on S\&P 500 cash index has been provided by The Futures Industry Institute (Washington, DC). 
arbitrage opportunities and this is an undesirable feature for obtaining a fair price. Thus, for instance, arbitrage would be possible within a portfolio containing bonds and stock whose strategy at time $t$ is buying (or selling) stock shares when $\mu+V(t)$ is greater (or lower) than the risk-free bond rate (Harrison et al (1984)).

In our case, however, the problem is that in the practice the return velocity $V(t)$ is nontradable and its evolution is ignored. In other words, in real markets the observed asset dynamics does not show any trace of the velocity variable田. This feature allows us to perform a projection of the two-dimensional diffusion process $[S(t), V(t)]$ onto a one-dimensional equivalent process $\bar{S}(t)$ independent of the velocity $V$. We will show in this section that the projected process $\bar{S}(t)$, which is equal to the actual price $S(t)$ in mean square sense, obeys the following one-dimensional SDE

$$
\frac{d \bar{S}(t)}{\bar{S}(t)}=[\mu+\dot{\kappa}(T-t) / 2] d t+\sqrt{\dot{\kappa}(T-t)} d W(t)
$$

where $\kappa(t)$ is given by Eq. (11), and the dot denotes time derivative. Therefore, the price given by Eq. (14) is driven by a noise of unbounded variation, the Wiener process, and the Harrison et al (1984) results do not apply. In consequence, the O-U projected process is still a suitable starting point for option pricing since it does not permit arbitrage.

\subsection{Derivation of the one-dimensional $S D E$}

Note that the dynamics of the return $R(t)=\ln \left[S(t) / S_{0}\right]$ is given by the second-order SDE (17) which includes the stochastic evolution of the velocity $V(t)$. Let us now obtain a first-order SDE describing the price dynamics when velocity $V(t)$ has been eliminated.

The starting point of our derivation is the marginal conditional density $p\left(R, t \mid R_{0}, t_{0} ; V_{0}\right)$. This density is given by Eq. (A.14) of Appendix A and when $t_{0} \neq 0$ it reads

$$
p\left(R, t \mid R_{0}, t_{0} ; V_{0}\right)=\frac{1}{\sqrt{2 \pi K_{11}\left(t-t_{0}\right)}} \exp \left\{-\frac{\left[R-R_{0}-m\left(t-t_{0}, V_{0}\right)\right]^{2}}{2 K_{11}\left(t-t_{0}\right)}\right\}
$$

where $m\left(t, V_{0}\right)$ and $K_{11}(t)$ are given by Eqs. (8) and (9). Note that $p\left(R, t \mid R_{0}, t_{0} ; V_{0}\right)$ is the solution of the following partial differential equation

$$
\frac{\partial p}{\partial t_{0}}=-\left[\mu+V_{0} e^{-\left(t-t_{0}\right) / \tau}\right] \frac{\partial p}{\partial R_{0}}-\frac{\sigma^{2}}{2}\left[1-e^{-\left(t-t_{0}\right) / \tau}\right]^{2} \frac{\partial^{2} p}{\partial R_{0}^{2}},
$$

with the final condition $p\left(R, t \mid R_{0}, t ; V_{0}\right)=\delta\left(R-R_{0}\right)$. Observe that the Eq. (16) is a backward Fokker-Planck equation whose drift, $\mu+V_{0} \exp \left[-\left(t-t_{0}\right) / \tau\right]$, and diffusion coefficient, $\frac{1}{2} \sigma^{2}\left[1-\exp \left[-\left(t-t_{0}\right) / \tau\right]\right]^{2}$, are both functions of $t-t_{0}$. As is well-known,

$\sharp$ Indeed, knowing $V(t)$ would imply knowing the value of the return $R(t)$ at two different times, since

$$
V(t)=\lim _{\epsilon \rightarrow 0^{+}} \frac{R(t)-R(t-\epsilon)}{\epsilon}-\mu .
$$

Obviously, this operation is not performed by traders who only manage portfolios at time $t$ based on prices at $t$ and not at any earlier time. 
there exists a direct relation between the Fokker-Planck equation and the SDE governing the process (Gardiner (1985)). In our case, the corresponding SDE is

$$
d R\left(t_{0}\right)=\left[\mu+V_{0} e^{-\left(t-t_{0}\right) / \tau}\right] d t_{0}+\sigma\left[1-e^{-\left(t-t_{0}\right) / \tau}\right] d W\left(t_{0}\right),
$$

and its formal solution is

$$
\begin{aligned}
R(t)=R\left(t_{0}\right)+\mu\left(t-t_{0}\right) & +V_{0} \tau\left[1-e^{-\left(t-t_{0}\right) / \tau}\right] \\
& +\sigma \int_{t_{0}}^{t}\left[1-e^{-\left(t-t_{1}\right) / \tau}\right] d W\left(t_{1}\right) .
\end{aligned}
$$

\subsection{Equality of processes in mean square sense}

To avoid confusion, let $\bar{R}(t)$ be the solution of the first-order SDE (17), i.e., $\bar{R}(t)$ is the projected process given by Eq. (18). And let $R(t)$ be the solution of the second-order SDE (7) where the dynamics of the velocity is still taken into account. Thus, $R(t)$ is explicitly given by Eq. (A.1) of Appendix A.

We will now prove that $\bar{R}(t)$ and $R(t)$ are equal in mean square sense. That is:

$$
E\left[(R(t)-\bar{R}(t))^{2}\right]=0, \quad \text { for any time } t .
$$

In effect, from Eq. (18) and assuming, without loss of generality, that $t_{0}=0$ and $\bar{R}\left(t_{0}\right)=0$ we have

$$
\bar{R}(t)=\mu t+V_{0} \tau\left(1-e^{-t / \tau}\right)+\int_{0}^{t}\left[1-e^{-\left(t-t_{1}\right) / \tau}\right] \xi\left(t_{1}\right) d t_{1}
$$

where $\xi\left(t_{1}\right)=d W\left(t_{1}\right) / d t_{1}$ is the Gaussian white noise. On the other hand, from Eq. (A.1) we write

$$
R(t)=\mu t+V_{0} \tau\left(1-e^{-t / \tau}\right)+\frac{\sigma}{\tau} \int_{0}^{t} d t^{\prime} \int_{0}^{t^{\prime}} e^{-\left(t^{\prime}-t^{\prime \prime}\right) / \tau} \xi\left(t^{\prime \prime}\right) d t^{\prime \prime} .
$$

Therefore,

$$
\begin{aligned}
E\left[(R(t)-\bar{R}(t))^{2}\right] & =2 K_{11}(t) \\
& -\frac{2 \sigma^{2}}{\tau} \int_{0}^{t} d t^{\prime} \int_{0}^{t^{\prime}} d t^{\prime \prime} e^{-\left(t^{\prime}-t^{\prime \prime}\right) / \tau} \int_{0}^{t} d t_{1}\left[1-e^{-\left(t-t_{1}\right) / \tau}\right] E\left[\xi\left(t_{1}\right) \xi\left(t^{\prime \prime}\right)\right],
\end{aligned}
$$

where $K_{11}(t)$ is given by Eq. (9). Taking into account that

$$
E\left[\xi\left(t^{\prime}\right) \xi(t)\right]=\delta\left(t^{\prime}-t\right),
$$

we have

$$
E\left[(R(t)-\bar{R}(t))^{2}\right]=2 K_{11}(t)-\frac{2 \sigma^{2}}{\tau} \int_{0}^{t} d t^{\prime} \int_{0}^{t^{\prime}} d t^{\prime \prime} e^{-\left(t^{\prime}-t^{\prime \prime}\right) / \tau}\left[1-e^{-\left(t-t^{\prime \prime}\right) / \tau}\right] .
$$

However, (see Eq. (9))

$$
\frac{\sigma^{2}}{\tau} \int_{0}^{t} d t^{\prime} \int_{0}^{t^{\prime}} d t^{\prime \prime} e^{-\left(t^{\prime}-t^{\prime \prime}\right) / \tau}\left[1-e^{-\left(t-t^{\prime \prime}\right) / \tau}\right]=K_{11}(t) .
$$

Hence,

$$
E\left[(R(t)-\bar{R}(t))^{2}\right]=0,
$$

and $R(t)$ is equal to $\bar{R}(t)$ in mean square sense. 


\subsection{The projected process when the initial velocity is in the stationary regime}

As we have mentioned, we are mainly interested in representing the asset dynamics when the initial velocity $V_{0}$ is random and distributed according to the stationary pdf (10). We have shown in Section 2 that this basically implies the replacement of $K_{11}(t)$ by $\kappa(t)$. In such a case, the SDE for $R(t)$ readst

$$
d R(t)=\mu d t+\sqrt{\dot{\kappa}(T-t)} d W(t),
$$

where $\kappa(t)$ is given by Eq. (11) and the dot denotes time derivative, that is

$$
\dot{\kappa}(t)=\sigma^{2}\left(1-e^{-t / \tau}\right) .
$$

We need the Itô lemma given in Appendix B for deriving the SDE for the stock $S$. Thus, according to Eq. (B.6), the effective dynamics for $S=S_{0} e^{R}$ is

$$
\frac{d S(t)}{S(t)}=[\mu+\dot{\kappa}(T-t) / 2] d t+\sqrt{\dot{\kappa}(T-t)} d W(t) .
$$

In this way, we have projected the two-dimensional O-U process $(S, V)$ onto a onedimensional price process which is a Wiener process with time varying drift and volatility. We also note that we need to specify the final condition of the process because the volatility $\sqrt{\dot{\kappa}}$ is a function of the time to maturity $T-t$, and this implies that the projected asset model depends on each particular contract.

\section{The option price on the projected process}

In this section we will present a generalization of the Black-Scholes theory assuming that underlying price is driven by the $\mathrm{O}-\mathrm{U}$ process. We therefore eliminate the efficient market hypothesis but retain the other two requirements of the original B-S theory: the absence of arbitrage and the existence of a riskless strategy.

We invoke the standard theoretical restrictions -continuos trading without transaction costs and dividends- and apply the original B-S method taking into account that the underlying asset is not driven by white noise but by colored noise modelled as an $\mathrm{O}-\mathrm{U}$ process.

The starting point of B-S option pricing is a portfolio which contains certain amounts of shares, calls and bonds. In this context, B-S hedging is only able to remove the call risk that comes from stock fluctuations. Therefore, we need to start from the effective one-dimensional market dynamics given by Eq. (23) since otherwise we would not be able to remove risk fluctuations arising from $d W(t)$. These fluctuations are only explicitly given in the projected SDE for the stock (see Section 5.1 for a deeper discussion on this point).

†† Since $R(t)$ and $\bar{R}(t)$ are equal in mean square sense we will drop the bar on $\bar{R}$ as long as there is no confusion. Thus, we will use $R$ for the projected process as well. 


\subsection{Black-Scholes option pricing with the equivalent one-dimensional SDE}

As we have proved in Section 3, there exists an effective one-dimensional diffusion which describes the $\mathrm{O}-\mathrm{U}$ process (21)-(3). Assuming that the effective one-dimensional price dynamics is given by Eq. (23), it is quite straightforward to derive the European call option price within the original B-S method. Following Merton (1973b) we define a portfolio compounded by a certain amount $\Delta$ of shares at price $S$, a quantity of bonds $\Phi$, and a number $\Psi$ of calls with price $C$, maturity time $T$ and strike price $K$. We assume that short-selling is allowed and thus the value $P$ of the portfolio is written

$$
P=\Psi C-\Delta S-\Phi B,
$$

where the bond price $B$ evolves according to the risk-free interest rate ratio $r$. That is

$$
d B=r B d t .
$$

The portfolio is required to obey the net-zero investment hypothesis, which means $P=0$ for any time $t$ (Merton (1973b)). Hence,

$$
C=\delta S+\phi B
$$

where $\delta=\Delta / \Psi$ and $\phi=\Phi / \Psi$ are, respectively, the number of shares per call and the number of bonds per call. Due to the nonanticipating character of $\delta$ and $\phi$ we have (Björk (1998))

$$
d C=\delta d S+\phi d B .
$$

On the other hand, assuming that the market dynamics is described by Eq. (23), the differential of the call also reads

$$
d C(S, t)=C_{t} d t+C_{S} d S+\frac{1}{2} \dot{\kappa}(T-t) S^{2} C_{S S} d t,
$$

where we have used the Itô lemma as expressed by Eq. (B.7) of the Appendix B. From Eqs. (27)-(28) and (26) we get

$$
\left[C_{t}+\frac{1}{2} \dot{\kappa}(T-t) S^{2} C_{S S}+r \delta S-r C\right] d t=\left[\delta-C_{S}\right] d S .
$$

Now the B-S delta hedging, $\delta=C_{S}$, removes any random uncertainty in the option price. The partial differential equation for $C(S, t)$ then reads

$$
C_{t}=r C-r S C_{S}-\frac{1}{2} \dot{\kappa}(T-t) S^{2} C_{S S} .
$$

We note that the delta hedging is able to remove risk because we have projected the two-dimensional SDE (目)-(政) onto the one-dimensional process. In this way, we directly relate the differential of the stock $d S(t)$ to the random fluctuations of the Wiener process $d W(t)$ (see Eq. (23)). Without this projection, the B-S hedging is useless and the random fluctuations persist in the B-S portfolio. We will further discuss this situation in Section 5.1 . 


\subsection{The price of the European call}

For the European call, Eq. (29) has to be solved with the following "final condition" at maturity time $T$

$$
C(S, T)=\max [S(T)-K, 0],
$$

where $S(T)$ is the underlying price at maturity and $K$ is the strike price. The solution to Eq. (29) subject to Eq. (30) is a type of solution perfectly known in the literature (see, for instance, Hull (2000)). Thus, our final price is

$$
C_{O U}(S, t)=S N\left(d_{1}^{O U}\right)-K e^{-r(T-t)} N\left(d_{2}^{O U}\right),
$$

where

$$
N(z)=(1 / \sqrt{2 \pi}) \int_{-\infty}^{z} e^{-x^{2} / 2} d x
$$

is the probability integral, and

$$
\begin{aligned}
& d_{1}^{O U}=\frac{\ln (S / K)+r(T-t)+\kappa(T-t) / 2}{\sqrt{\kappa(T-t)}}, \\
& d_{2}^{O U}=d_{1}^{O U}-\sqrt{\kappa(T-t)},
\end{aligned}
$$

with $\kappa(t)$ given by Eq. (11).

Equation (31) constitutes the key result of the paper. Note that, when $\tau=0$, the variance becomes $\kappa(t)=\sigma^{2} t$ and the price in Eq. (31) reduces to the Black-Scholes price:

$$
C_{B S}(S, t)=S N\left(d_{1}^{B S}\right)-K e^{-r(T-t)} N\left(d_{2}^{B S}\right),
$$

where $d_{1,2}^{B S}$ have the form of Eqs. (32)-(33) with $\kappa(T-t)$ replaced by $\sigma^{2}(T-t)$. Therefore, the $\mathrm{O}-\mathrm{U}$ price in Eq. (31) has the same functional form as B-S price in Eq. (34) when $\sigma^{2} t$ is replaced by $\kappa(t)$.

In the opposite case, $\tau=\infty$, where there is no random noise but a deterministic and constant driving force (in our case it is zero), Eq. (31) reduces to the deterministic price

$$
C_{d}(S, t)=\max \left[S-K e^{-r(T-t)}, 0\right] .
$$

We will now prove that $C_{O U}$ is an intermediate price between B-S price and the deterministic price (see Fig. 2)

$$
C_{d}(S, t) \leq C_{O U}(S, t) \leq C_{B S}(S, t),
$$

for all $S$ and $0 \leq t \leq T$. In order to prove this it suffices to show that $C_{O U}$ is a monotone decreasing function of the correlation time $\tau$, since in such a case

$$
C_{O U}(\tau=\infty) \leq C_{O U}(\tau) \leq C_{O U}(\tau=0)
$$

However, $C_{O U}(\tau=\infty)=C_{d}$ and $C_{O U}(\tau=0)=C_{B S}$, which leads to Eq. (36). Let us thus show that $C_{O U}$ is a decreasing function of $\tau$ for $0 \leq t \leq T$ and all $S$. Define a function $\alpha$ as the derivative

$$
\alpha=\frac{\partial C_{O U}}{\partial \tau} .
$$






Figure 2. Relative call price $C / S$ as a function of $S / K$ for a given time to expiration $T-t=5$ days. The solid line represents the O-U call price with $\tau=1$ day and the dashed line is the B-S price. The dotted line is the deterministic price. In this figure the annual risk-free interest rate $r=5 \%$, and the annual volatility $\sigma=30 \%$.

Since the $\tau$ dependence in $C_{O U}$ is a consequence of the variance $\kappa(t, \tau)$, we have

$$
\alpha=\frac{\sigma}{2 \kappa(T-t, \tau)} \frac{\partial \kappa(T-t, \tau)}{\partial \tau} \mathcal{V}_{O U},
$$

where $\mathcal{V}_{O U}=\partial C_{O U} / \partial \sigma$ (see Section 7). But

$$
\frac{\partial \kappa(T-t, \tau)}{\partial \tau}=-\sigma^{2}\left[1-(1+(T-t) / \tau) e^{-(T-t) / \tau}\right] \leq 0,
$$

for $0 \leq t \leq T$ which is seen to be non positive. From Eq. (67) below we see that $\mathcal{V}_{O U} \geq 0$ for all $S$ and $0 \leq t \leq T$. Hence, $\alpha \leq 0$ which proves Eq. (36). In Fig. 3 we plot the option price $C$ as a function of the correlation time $\tau$ and for three different values of the moneyness $S / K$. This figure clearly shows that $C$ is a monotone decreasing function of $\tau$.

Therefore, the assumption of uncorrelated underlying assets ( $B$-S case) overprices any call option. This confirms the intuition understanding that correlation implies more predictability and therefore less risk and, finally, a lower price for the option. In fact, we can easily quantify this overprice by evaluating the relative difference

$$
D=\left(C_{B S}-C_{O U}\right) / C_{B S} .
$$

Figure 1 shows the ratio $D(S, t)$, for a fixed time to expiration, plotted as a function of the moneyness, $S / K$, and for different values of correlation time $\tau$. We see there 


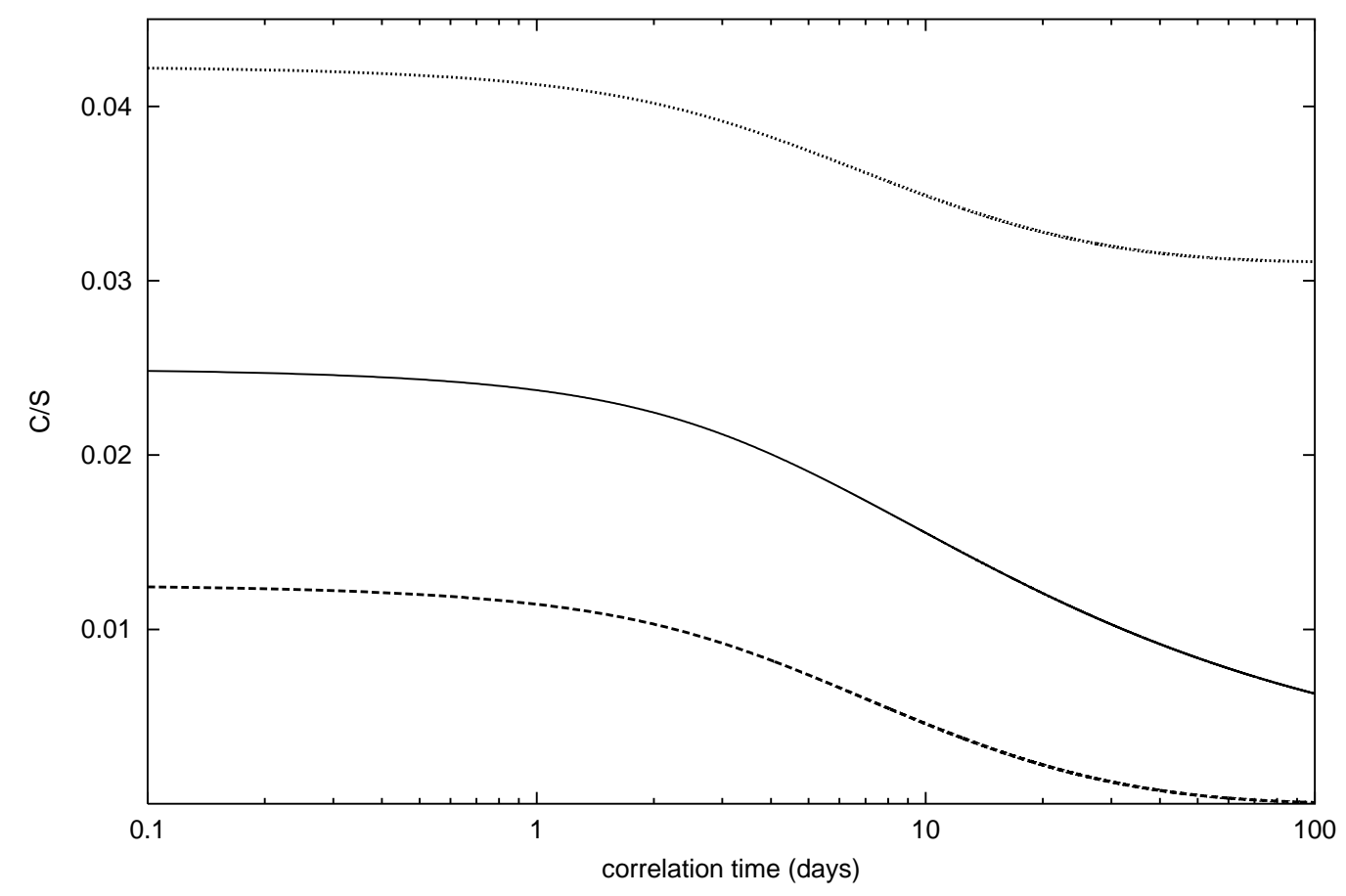

Figure 3. Relative call price $C / S$ as a function of $\tau$ for a given time to expiration $T-t=10$ days. The solid line represents the call price with $S / K=1$ (ATM case). The dotted line is the call price when $S / K=1.03$ (ITM case). The dashed line represents an OTM case when $S / K=0.97$. We clearly see that $C$ is a monotone decreasing function of $\tau$ having its maximum value when $\tau=0$ (B-S case) and its minimum when $\tau \rightarrow \infty$ (deterministic price). The annual risk-free interest rate and the annual volatility are as in Fig. 2.

that the ratio $D$ is very sensitive to whether the call is in the money (ITM), out of the money (OTM) or at the money (ATM). The biggest difference between prices occurs in the case of OTM options. This is true because when $S / K<1$, both $C_{B S}$ and $C_{O U}$ are small but $C_{B S} \gg C_{O U}$ (see Fig. 目). Depending on the value of correlation time $\tau$ this implies that $D$ is approximately equal to 1 .

Another interesting point is the behavior of $D$ as a function of the expiration time $T-t$. In this case, $D$ behaves quite differently depending on whether the call is in, out, or at the money. This behavior is evident in Figs. 5 and 6 . Figure 5 shows $D(S, t)$ as a function of expiration time $T-t$ for an OTM option $(S / K=0.95)$ and the ATM option $(S / K=1.00)$ and for two different values (1 and 5 days) of the correlation time. Note that B-S notably overprices the option, particularly in the OTM case. In Fig. 6 we show plots of $D(S, t)$ as a function of $t$ for an ITM option $(S / K=1.05)$. This exhibits completely different behavior since the B-S overprice is considerably less (no more than 7\%). Moreover, contrary to the ATM and OTM cases, the relative difference $D(S, t)$ is a non monotone function of $T-t$, having a maximum value around one or two weeks before maturity. Although perhaps the most striking and interesting feature is the 


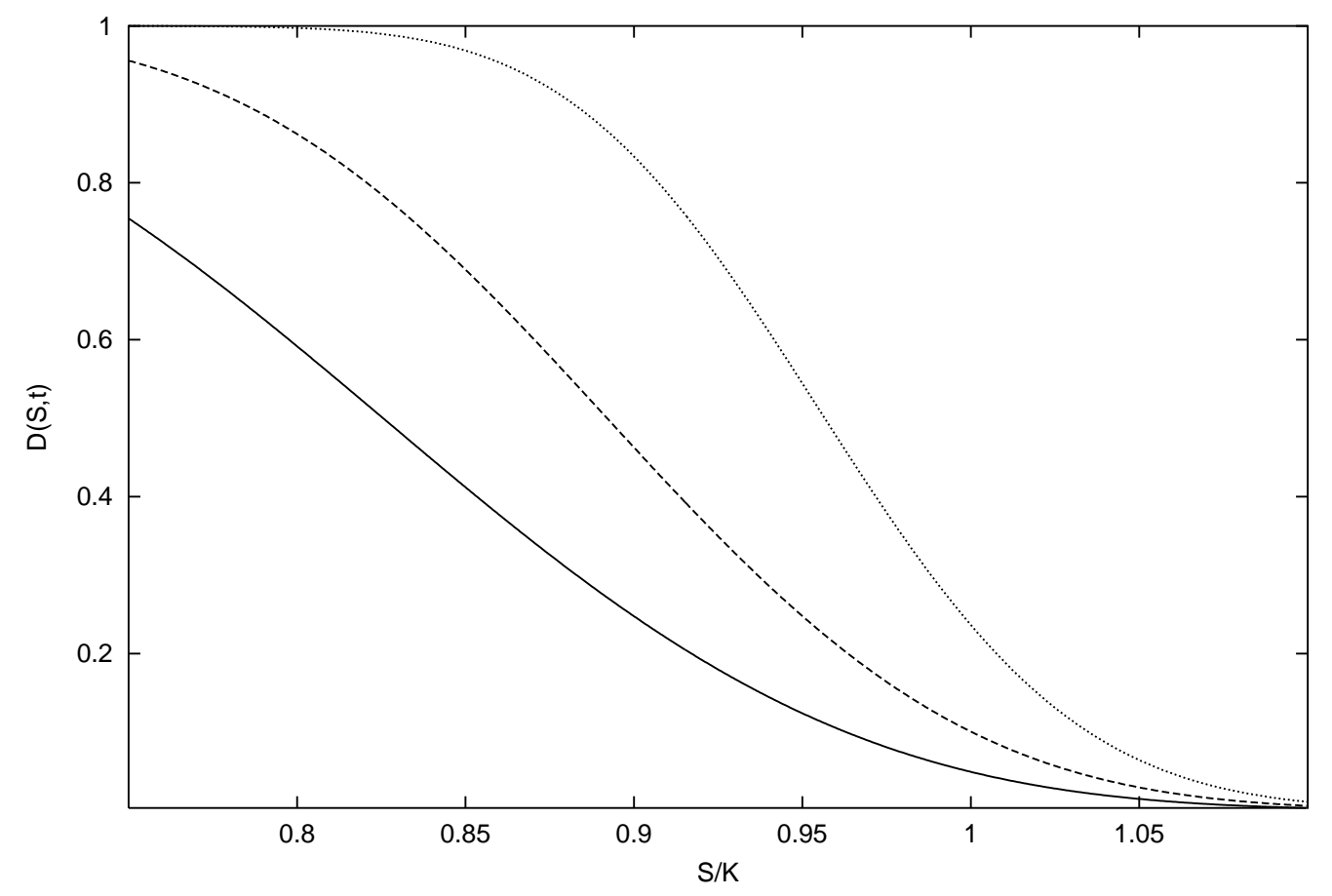

Figure 4. $D(S, t)$ is plotted as a function of $S / K$ for $T-t=10$ days and $\tau=1$ day (solid line), $\tau=2$ days (dashed line) and $\tau=5$ days (dotted line). Other parameters used to generate the figure are $r=5 \%$ per annum and $\sigma=30 \%$ per annum.

persistence of the B-S overprice far from maturity regardless the value of the correlation time. This is clearly shown in Table 1 where we quantify the ratio $D$ in percentages for different values of moneyness, time to expiration and correlation time.

\section{An alternative derivation of the call price}

In this section and the next, we present two different and alternative derivations of the final call price $C_{O U}$. The first of these derivations is based on an extension of the B-S theory but now starting from the two-dimensional diffusion (21)-(3) and with a different portfolio than the usual one. A second derivation, briefly outlined in the next section, uses the equivalent martingale measure method. Both derivations arrive at the price formula (31), thus showing the consistency of the pricing methods.

We will first apply the original B-S method starting from the two-dimensional O-U process (2)-(酒) instead of the equivalent process (23). Unfortunately, this procedure yields a trivial expression for the price of the option (see below) and is therefore useless. To avoid this difficulty we will define a different portfolio which is the first step towards the generalization of both B-S equation and formula. 


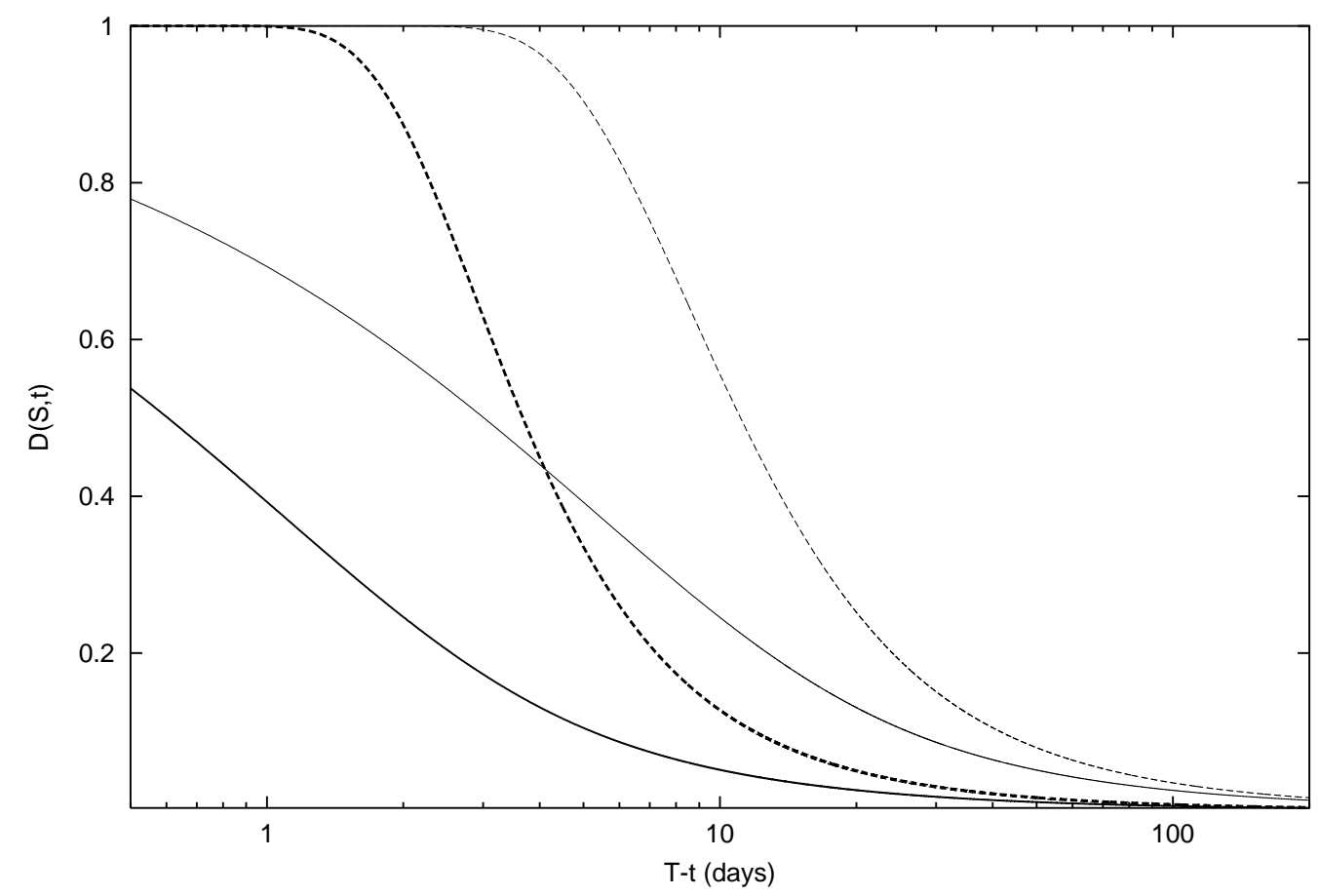

Figure 5. $D(S, t)$ is plotted as a function of $T-t$ (in logarithmic scale) for fixed values of moneyness. The solid lines represent ATM options, the thick line corresponds to $\tau=1$ day and the thin line corresponds to $\tau=5$ days. The dashed lines represent an OTM option with $S / K=0.95$, the thick line corresponds to $\tau=1$ day and the thin line corresponds to $\tau=5$ days ( $r$ and $\sigma$ as in Fig. 2).

\subsection{The Black-Scholes method for the two-dimensional O-U process}

We assume that market prices are driven by an $\mathrm{O}-\mathrm{U}$ process as shown in Eqs. (2)-(3) and that the portfolio is given by Eq. (24). That is, $C=\delta S+\phi B$ and

$$
d C=\delta d S+\phi d B .
$$

Let us now apply the original B-S method starting from the two-dimensional O-U process (2)-(3) instead of the equivalent process (23). Using the Itô lemma for a singular two-dimensional diffusion (see Appendix B),

$$
d C(S, V, t)=C_{S} d S+C_{V} d V+C_{t} d t+\frac{\sigma^{2}}{2 \tau^{2}} C_{V V} d t,
$$

and taking Eqs. (25) and (27) into account, we write

$$
\left[C_{t}+\frac{\sigma^{2}}{2 \tau} C_{V V}-r(C-S \delta)\right] d t+\left(C_{S}-\delta\right) d S+C_{V} d V=0
$$

Now the assumption of delta hedging $\delta=C_{S}$, turns this equation into

$$
\left[C_{t}-r\left(C-S C_{S}\right)+\frac{\sigma^{2}}{2 \tau} C_{V V}\right] d t+C_{V} d V=0 .
$$




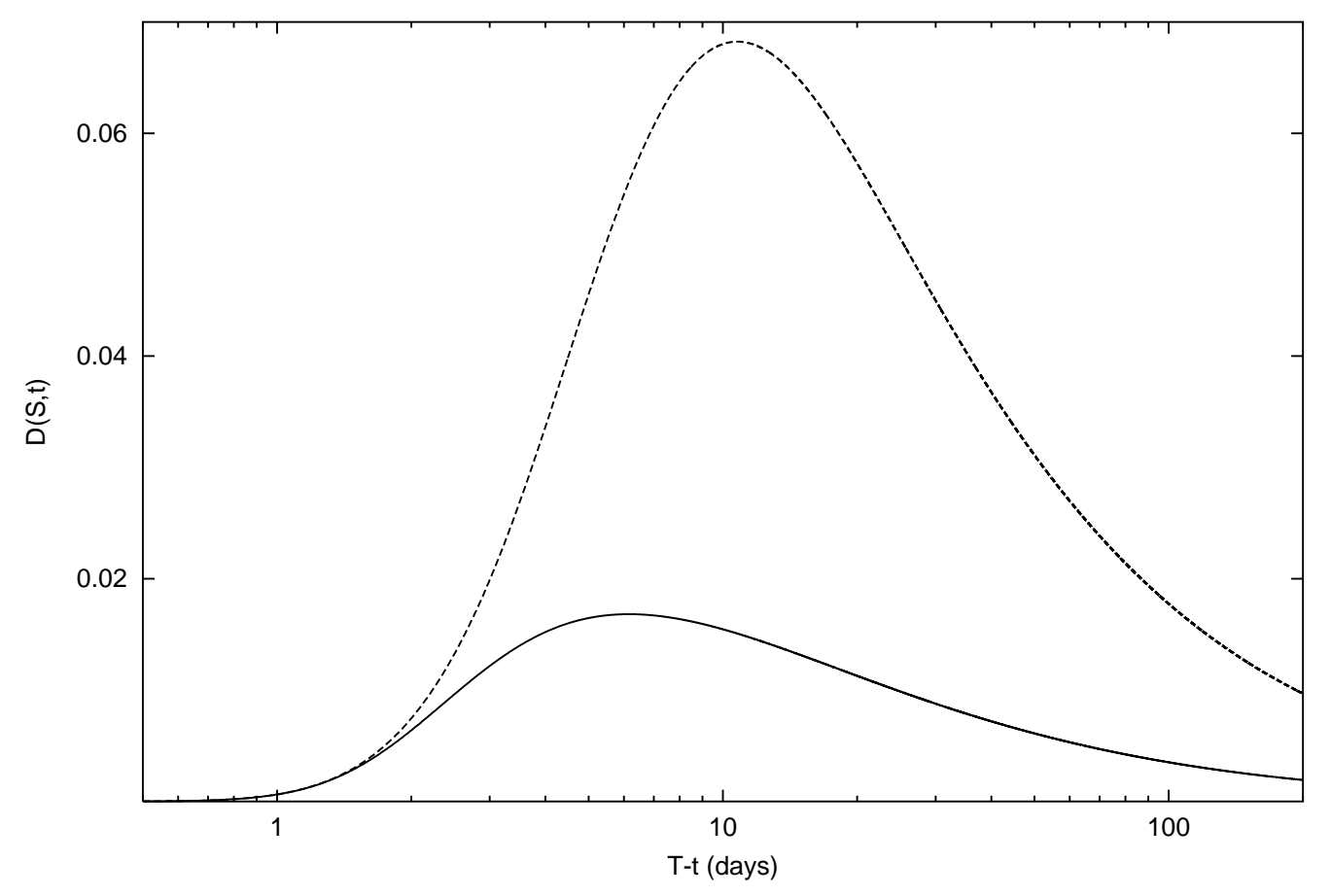

Figure 6. $D(S, t)$ is plotted as a function of the expiration time $T-t$ (in logarithmic scale) for an ITM option with $S / K=1.05$. The solid line corresponds to $\tau=1$ day and the dashed line to $\tau=5$ days ( $r$ and $\sigma$ as in Fig. 2).

Equation (39) is still random due to the term with $d V$ representing velocity fluctuations (see Eq. (3)). In consequence, Black-Scholes delta hedging is incomplete since it is not able to remove risk. In this situation, the only way to derive a risk-free partial differential equation for the call price is to assume that the call is independent of velocity. Then, $C_{V}=0$ and Eq. (39) yields

$$
C_{t}+r S C_{S}-r C=0 .
$$

According to the final condition for the European call, $C(S, T)=\max [S(T)-K, 0]$, the call price is $C(S, t)=\max \left[S-K e^{-r(T-t)}, 0\right]$. Note that this is a useless expression because it gives a price for the option as if the underlying asset would have evolved deterministically like the risk-free bond without pricing the random evolution of the stock. In fact, there is no hint of randomness, measured by the volatility $\sigma$, in Eq. (40).

The main reason for the failure of B-S theory is the inappropriateness of B-S hedging for two-dimensional processes such as O-U price process (2)-(3) presumably diversifies away the risk associated with the differential of asset price $d S(t)$ given by Eq. (2). Nevertheless, what we have to hedge is the risk associated with $d V(t)$ given by Eq. (3), which contains the only source of randomness: the differential of the Wiener process $d W(t)$. All of this clearly shows the uselessness of the B-S delta hedging for the two-dimensional $\mathrm{O}-\mathrm{U}$ process. Note that we must relate in a direct way the

‡ A similar situation appears in the stochastic volatility models (Scott (1987)). 
Table 1. Relative call price differences in percentages. Values of $D \times 100$, where $D=\left(C_{B S}-C_{O U}\right) / C_{B S} . T-t$ is the expiration time in days. Correlation times $\tau$ are 1,2 , and 5 days. The rest of columns are divided in three blocks corresponding to a different values of the moneyness $S / K$. From left to right blocks represent the OTM, ATM, and ITM cases. Notice the importance and the persistence far from maturity of the relative differences in price ( $r$ and $\sigma$ as in Fig. 2).

\begin{tabular}{llllllllll}
\hline$T-t$ & \multicolumn{3}{c}{$S / K=0.95$} & \multicolumn{3}{c}{$S / K=1.00$} & \multicolumn{3}{c}{$S / K=1.05$} \\
& $\tau=1$ & 2 & 5 & $\tau=1$ & 2 & 5 & $\tau=1$ & 2 & 5 \\
\hline 1 & 99.9 & 100 & 100 & 39.3 & 53.8 & 69.3 & 0.1 & 0.1 & 0.1 \\
2 & 87.4 & 98.7 & 100 & 24.6 & 39.3 & 58.0 & 0.6 & 0.7 & 0.7 \\
3 & 62.9 & 88.3 & 99.5 & 17.3 & 30.5 & 50.1 & 1.2 & 1.7 & 2.0 \\
4 & 45.0 & 73.1 & 96.5 & 13.1 & 24.6 & 44.1 & 1.5 & 2.5 & 3.4 \\
5 & 33.5 & 59.4 & 90.4 & 10.5 & 20.4 & 39.2 & 1.6 & 2.9 & 4.6 \\
6 & 26.1 & 48.6 & 82.8 & 8.7 & 17.3 & 35.3 & 1.7 & 3.1 & 5.5 \\
7 & 21.0 & 40.4 & 75.1 & 7.4 & 14.9 & 31.9 & 1.7 & 3.2 & 6.1 \\
8 & 17.4 & 34.1 & 67.9 & 6.4 & 13.1 & 29.1 & 1.6 & 3.2 & 6.5 \\
9 & 14.7 & 29.2 & 61.3 & 5.7 & 11.6 & 26.7 & 1.6 & 3.2 & 6.7 \\
& & & & & & & & & \\
10 & 12.7 & 25.4 & 55.6 & 5.1 & 10.4 & 24.5 & 1.5 & 3.1 & 6.8 \\
20 & 5.0 & 10.0 & 25.2 & 2.5 & 5.1 & 13.1 & 1.1 & 2.3 & 5.7 \\
30 & 2.9 & 5.9 & 15.1 & 1.7 & 3.4 & 8.6 & 0.9 & 1.8 & 4.5 \\
40 & 2.0 & 4.1 & 10.5 & 1.2 & 2.5 & 6.4 & 0.7 & 1.4 & 3.7 \\
50 & 1.6 & 3.1 & 7.9 & 1.0 & 2.0 & 5.1 & 0.6 & 1.2 & 3.1 \\
& & & & & & & & & \\
100 & 0.7 & 1.4 & 3.4 & 0.5 & 1.0 & 2.5 & 0.4 & 0.7 & 1.8 \\
150 & 0.4 & 0.8 & 2.2 & 0.3 & 0.7 & 1.6 & 0.2 & 0.5 & 1.3 \\
200 & 0.3 & 0.6 & 1.5 & 0.2 & 0.5 & 1.2 & 0.2 & 0.4 & 1.0 \\
& 0.2 & 0.5 & 1.2 & 0.2 & 0.4 & 1.0 & 0.2 & 0.3 & 0.8 \\
\hline 50 & & & & & & & & &
\end{tabular}

differential $d S(t)$ with the random differential $d W(t)$, otherwise we will not be able to remove risk. This is indeed the case of the projected process (23) which leads to the European call price, Eq. (31). However, if we do not want to project the process and maintain the two-dimensional formulation (2)-(3) we have to evaluate the option price from a different portfolio. We will do it next by defining a new portfolio which will allow us to preserve the complet market hypothesis and remove the random component $d W(t)$.

\subsection{The option pricing method with a modified portfolio}

We present a new portfolio in a complete but not efficient market. The market is still assumed to be complete, in other words, there exists a portfolio with assets to 
eliminate financial risk. However, we relax the efficient market hypothesis by including the correlated $\mathrm{O}-\mathrm{U}$ process as noise for the underlying price dynamics.

Now, our portfolio is compounded by a number of calls $\Psi$ with maturity $T$ and strike $K$, a quantity of bonds $\Phi$, and another number of "secondary calls" $\Psi^{\prime}$, on the same asset, but with a different strike $K^{\prime}$ and, eventually, different payoff or maturity time. Note that in the new portfolio there are no shares of the underlying asset. Thus, instead of Eq. (24), we have

$$
P=\Psi C-\Psi^{\prime} C^{\prime}-\Phi B .
$$

After assuming the net-zero investment, we obtain

$$
C=\phi B+\psi C^{\prime},
$$

where $\phi \equiv \Phi / \Psi$ is the number of bonds per call, and $\psi \equiv \Psi^{\prime} / \Psi$ is the number of secondary calls per call. We proceed as before, thus the nonaticipating character of $\phi$ and $\psi$ allows us to write

$$
d C=\phi d B+\psi d C^{\prime}
$$

and, after using Itô lemma (38) for both $d C$ and $d C^{\prime}$, some simple manipulations yield

$$
\begin{aligned}
& {\left[\left(C_{t}+\frac{\sigma^{2}}{2 \tau} C_{V V}-r C+(\mu+V) S C_{S}\right)\right.} \\
& \left.\quad-\psi\left(C_{t}^{\prime}+\frac{\sigma^{2}}{2 \tau} C_{V V}^{\prime}-r C^{\prime}+(\mu+V) S C_{S}^{\prime}\right)\right] d t=\left(\psi C_{V}^{\prime}-C_{V}\right) d V .
\end{aligned}
$$

This equation can be transformed to a deterministic one by equating to zero the term multiplying the random differential $d V(t)$ given by Eq. (3). This, in turn, will determine the investor strategy giving the relative number of secondary calls to be held. Thus, instead of B-S delta hedging, we will have the "psi hedging":

$$
\psi=\frac{C_{V}}{C_{V}^{\prime}} \text {. }
$$

Then

$$
\begin{aligned}
\frac{1}{C_{V}}\left[C_{t}+\frac{\sigma^{2}}{2 \tau} C_{V V}\right. & \left.-r C+(\mu+V) S C_{S}\right] \\
& =\frac{1}{C_{V}^{\prime}}\left[C_{t}^{\prime}+\frac{\sigma^{2}}{2 \tau} C_{V V}^{\prime}-r C^{\prime}+(\mu+V) S C_{S}^{\prime}\right] .
\end{aligned}
$$

This equation proves, as otherwise expected, that the call has the same partial differential equation independent of its maturity and strike. This has been suggested in a more theoretical setting for any derivative on the same asset (Björk (1998)).

On the other hand, the two options $C$ and $C^{\prime}$ have different strikes. Then, analogously to the separation of variable method used in mathematics (Mynt-U (1987)) and proceeding in a similar way to that used in the study of SV cases, both sides of Eq. (46) are assumed to be equal to an unknown function $\lambda(S, V, t)$ of the independent variables $S, V$, and $t$. We thus have

$$
C_{t}+\frac{\sigma^{2}}{2 \tau} C_{V V}+(\mu+V) S C_{S}-r C=\lambda C_{V} .
$$


In the stochastic volatility literature, the arbitrary function $\lambda(S, V, t)$ is known as the "risk premium" associated, in our case, with the return velocity (Scott (1987); Heston (1993)). In the Appendix $\mathrm{C}$ we show that the risk premium $\lambda$ is given by

$$
\lambda(S, V, t)=\frac{V}{\tau} .
$$

A substitution of Eq. (48) into Eq. (47) yields a closed partial differential for the call price $C(S, V, t)$ which is

$$
C_{t}+\frac{\sigma^{2}}{2 \tau} C_{V V}-\frac{V}{\tau} C_{V}+(\mu+V) S C_{S}-r C=0 .
$$

For the European call, Eq. (49) has to be solved with the "final condition" (30) at maturity time which is $C(S, V, T)=\max [S(T)-K, 0]$. The solution to Eq. (49) subject to Eq. (30) is given in Appendix D and reads

$$
C(S, V, t)=e^{-r(T-t)}\left[S e^{\beta(T-t, V)} N\left(z_{1}\right)-K N\left(z_{2}\right)\right],
$$

where $z_{1}=z_{1}(S, V, T-t), z_{2}=z_{2}(S, V, T-t)$ are given by Eq. (D.10) of Appendix D, and

$$
\beta(t, V)=m(t, V)+K_{11}(t) / 2,
$$

where $m(t, V)$ and $K_{11}(t)$ are given by Eqs. (8) and (9).

The option price (50) depends on both the price $S$ and the velocity $V$ of the underlying asset at time $t$, i.e., at the time at which the call is bought. They are therefore the initial variables of the problem. However, while the initial price $S$ is always known, the initial velocity $V$ is unknown. The velocity is thus assumed to be in the stationary regime so that its probability density function is as shown in Eq. (10). We therefore average over the unknown initial velocity and define $\bar{C}$ by

$$
\bar{C}(S, t) \equiv \int_{-\infty}^{\infty} C(S, V, t) p_{s t}(V) d V,
$$

and from Eqs. (10) and (50) we have

$$
\bar{C}(S, t)=e^{-r(T-t)}\left[S e^{\beta(T-t)} N\left(\bar{z}_{1}\right)-K N\left(\bar{z}_{2}\right)\right],
$$

where

$$
\beta(t)=\mu t+\kappa(t) / 2,
$$

$\kappa(t)$ is the variance defined by Eq. (11), and $\bar{z}_{1,2}$ are given by Eq. (D.11) of Appendix D. As mentioned above, Eq. (52) cannot be our final price yet because it still depends on the mean return rate $\mu$. This rate could differ depending on whether $\mu$ is estimated by the seller or buyer of the option and thereofore, in Eq. (52), there are hidden arbitrage opportunities.

Therefore, we must proceed in a similar way as in the martingale option pricing theory of Eq. (62) and define the final call price, $C_{O U}(S, t)$, as price $\bar{C}$ when $\beta(t)$ is replaced by $r t$. That is:

$$
\left.C_{O U}(S, t) \equiv \bar{C}(S, t)\right|_{\beta(t) \rightarrow r t},
$$

and this price completely agrees with the one derived in Section 3 (see Eq. (31)). 


\subsection{The projected process and the modified portfolio}

Suppose we start from the modified portfolio (42) but assuming that the share price is given by the projected process (23) instead of the two-dimensional O-U process (22)-(3). In this case, one can obtain the same option price as before (cf. Eq. (31)). However, the hedging strategy will be given by the following function

$$
\psi(S, t)=\frac{C_{S}}{C_{S}^{\prime}} .
$$

Let us prove this. We start from Eq. (43):

$$
d C=\phi d B+\psi d C^{\prime},
$$

Now, instead of Eq. (44) we have (see Itô lemma (28))

$$
\begin{gathered}
{\left[\left(C_{t}+\frac{1}{2} \dot{\kappa}(T-t) C_{S S}-r C\right)-\psi\left(C_{t}^{\prime}+\frac{1}{2} \dot{\kappa}(T-t) C_{S S}^{\prime}-r C^{\prime}\right)\right] d t} \\
=\left(\psi C_{S}^{\prime}-C_{S}\right) d S .
\end{gathered}
$$

And the removal of risk implies Eq. (55). The psi hedging given by Eq. (55) is equivalent to the psi hedging defined in Eq. (45) although now it is represented in terms of the final price $C_{O U}(S, t)$ instead of the intermediate price $C(S, V, t)$. Substituting Eq. (55) into Eq. (56) and reasoning along the same lines as above (see Eq. (47)) we obtain

$$
C_{t}+\frac{1}{2} \dot{\kappa}(T-t) S^{2} C_{S S}-r C=\lambda C_{S},
$$

where $\lambda=\lambda(S, t)$ is the "risk premium" for the effective process which is now obviously independent of the velocity $V$. Combining Eqs. (23), (28) and (57), we get

$$
\begin{aligned}
d C(S, t)=\left\{r C+\left[\frac{\lambda}{S}+\mu+\right.\right. & \left.\left.\frac{1}{2} \dot{\kappa}(T-t)\right] S C_{S}\right\} d t \\
& +\sqrt{\dot{\kappa}(T-t)} S C_{S} d W(t) .
\end{aligned}
$$

Hence, the conditional expected value of $d C$ reads

$$
E[d C \mid C]=\left\{r C+\left[\frac{\lambda}{S}+\mu+\frac{1}{2} \dot{\kappa}(T-t)\right] S C_{S}\right\} d t,
$$

but the equilibrium of the market implies that $E[d C \mid C]=r C d t$. Therefore,

$$
\lambda=-S\left[\mu+\frac{1}{2} \dot{\kappa}(T-t)\right],
$$

and Eq. (57) reads

$$
C_{t}+\frac{1}{2} \dot{\kappa}(T-t) S^{2} C_{S S}-r C+\left[\mu+\frac{1}{2} \dot{\kappa}(T-t)\right] S C_{S}=0,
$$

Finally, the absence of arbitrage opportunities requires the replacement (see Eq. (53))

$$
\mu+\dot{\kappa}(T-t) / 2 \longrightarrow r .
$$

Thus, the option price equation is

$$
C_{t}=r C-r S C_{S}-\frac{1}{2} \dot{\kappa}(T-t) S^{2} C_{S S},
$$


which agrees with Eq. (29).

Note that both procedures, the original B-S method presented in Section 3 and our method, result in the same partial differential equation for the call price. However, each method uses a different hedging strategy because they start from a different portfolio.

\section{The call price by the equivalent martingale measure method}

As was shown by Harrison and Kreps (1979) and Harrison and Pliska (1981), the B-S option price can also be found using martingale methods. This is a shorter, although more abstract way, to derive an expression for the call price. The main advantage is that one only needs to know the probability density function governing market evolution which, in turn, allows one to obtain an option price in situations where B-S assumptions are not applicable. The drawback is that one is not sure of whether the price obtained by martingale methods is the fair price of the call because of the omission of arbitrage and hedging.

We will now show that, in the present case, the price obtained by martingale methods completely agrees with our extended B-S price (31). The equivalent martingale measure theory imposes the condition that, in a "risk-neutral world", the stock price $S(t)$ evolves, on average, as a riskless bond (Harrison and Pliska (1981)).

Let $p^{*}\left(S, t \mid S_{0}, t_{0}\right)$ be the equivalent martingale measure associated with asset price $S(t)$ conditioned on $S\left(t_{0}\right)=S_{0}$. Define the martingale conditional expected value

$$
E^{*}\left[S(t) \mid S_{0}\right]=\int_{0}^{\infty} S p^{*}\left(S, t \mid S_{0}, t_{0}\right) d S .
$$

Then the risk-neutral assumption requires that

$$
E^{*}\left[S(t) \mid S_{0}\right]=S_{0} e^{r\left(t-t_{0}\right)},
$$

where $r$ is the constant spot interest rate. On the other hand,

$$
E\left[S(t) \mid S_{0}\right]=\int_{0}^{\infty} S p\left(S, t \mid S_{0}, t_{0}\right) d S .
$$

Assuming that the initial velocity is in the statioanry regime, the marginal density $p\left(S, t \mid S, t_{0}\right)$ is given by Eq. (A.16) of Appendix A. Therefore,

$$
E\left[S(t) \mid S_{0}\right]=S_{0} \exp \left[\beta\left(t-t_{0}\right)\right],
$$

where $\beta(t)=\mu t+\kappa(t) / 2$ with $\kappa(t)$ given by Eq. (11). We thus see that the equivalent martingale measure is accomplished by the replacement

$$
\beta(t) \longrightarrow r t .
$$

In consequence,

$$
\begin{aligned}
p^{*}\left(S, t \mid S_{0}, t_{0}\right)= & \frac{1}{S \sqrt{2 \pi \kappa\left(t-t_{0}\right)}} \\
& \times \exp \left\{-\frac{\left[\ln \left(S / S_{0}\right)-r\left(t-t_{0}\right)+\kappa\left(t-t_{0}\right) / 2\right]^{2}}{2 \kappa\left(t-t_{0}\right)}\right\},
\end{aligned}
$$


which is the so called "the risk-neutral pdf" for the stock price and it is a consequence of the absence of arbitrage demmand. Now, it is possible to express the price for the European call option by defining its value as the discounted expected gain due to holding the call. That is (Harrison and Pliska (1981)),

$$
\begin{aligned}
C^{*}(S, t) & =e^{-r(T-t)} E^{*}\{\max [S(T)-K, 0] \mid S(t)=S\} \\
& =e^{-r(T-t)} \int_{K}^{\infty}\left(S^{\prime}-K\right) p^{*}\left(S^{\prime}, T \mid S, t\right) d S^{\prime},
\end{aligned}
$$

and the final result for the call is obtained by calculating the expected value with the equivalent martingale measure defined in Eq. (63). The martingale price agrees exactly with our previous price in Eq. (31), $C^{*}(S, t)=C_{O U}(S, t)$. We can thus say that, in the $\mathrm{O}-\mathrm{U}$ case, both option pricing methods are completely equivalent although martingale theory does not require the construction of a portfolio and ignores any hedging strategy.

\section{Greeks and Hedging}

We briefly derive the Greeks for the O-U case. Since the O-U call price has the same functional form as the B-S price but replaces $\sigma^{2}(T-t)$ by $\kappa(T-t)$, the O-U Greeks will have the same functional form as B-S Greeks with the same replacement except for Vega, $\mathcal{V}=\partial C / \partial \sigma$, and $\theta=\partial C / \partial t$. Thus, for $\delta=\partial C / \partial S, \gamma=\partial^{2} C / \partial S^{2}$, and $\rho=\partial C / \partial r$, we have (Hull (2000))

$$
\begin{aligned}
\delta_{O U}=N\left(d_{1}^{O U}\right), \quad \gamma_{O U} & =\frac{e^{-\left(d_{1}^{O U}\right)^{2} / 2}}{S \sqrt{2 \pi \kappa(T-t)}}, \\
\rho_{O U} & =K(T-t) e^{-r(T-t)} N\left(d_{2}^{O U}\right) .
\end{aligned}
$$

Since $d_{1,2}^{O U} \geq d_{1,2}^{B S}$ for all $S$ and $t$ and $N(z)$ is a monotone increasing function, we see that $\delta_{O U} \geq \delta_{B S}$ and $\rho_{O U} \geq \rho_{B S}$. Hence, the O-U call price is more sensitive to changes in stock price and interest rate than the B-S price.

On the other hand, from Eq. (31) and taking into account the identity

$$
S N^{\prime}\left(d_{1}\right)-K e^{-r(T-t)} N^{\prime}\left(d_{2}\right)=0,
$$

we have

$$
\mathcal{V}_{O U}=(S / \sigma)[\kappa(T-t) / 2 \pi]^{1 / 2} e^{-\left(d_{1}^{O U}\right)^{2} / 2}
$$

and

$$
\theta_{O U}=-K e^{-r(T-t)}\left[r N\left(d_{2}^{O U}\right)+\frac{\sigma^{2}\left(1-e^{-(T-t) / \tau}\right)}{2 \sqrt{2 \pi \kappa(T-t)}} e^{-\left(d_{2}^{O U}\right)^{2} / 2}\right] .
$$

Since $d_{1}^{O U} \geq d_{1}^{B S}$, one can easily see that $\mathcal{V}_{O U} \leq \mathcal{V}_{B S}$ for all values of $S / K, T-t$ and $\tau$. Thus our correlated call price is less sensitive to any change of underlying volatility $\sigma$ than is the B-S price.

We conclude with the psi hedging. For the two-dimensional O-U case the hedging strategy is given by the function $\psi(S, V, t)$ specifying the number of secondary calls to be hold. However, the hedging given by Eq. (45) depends on the velocity $V$ and 


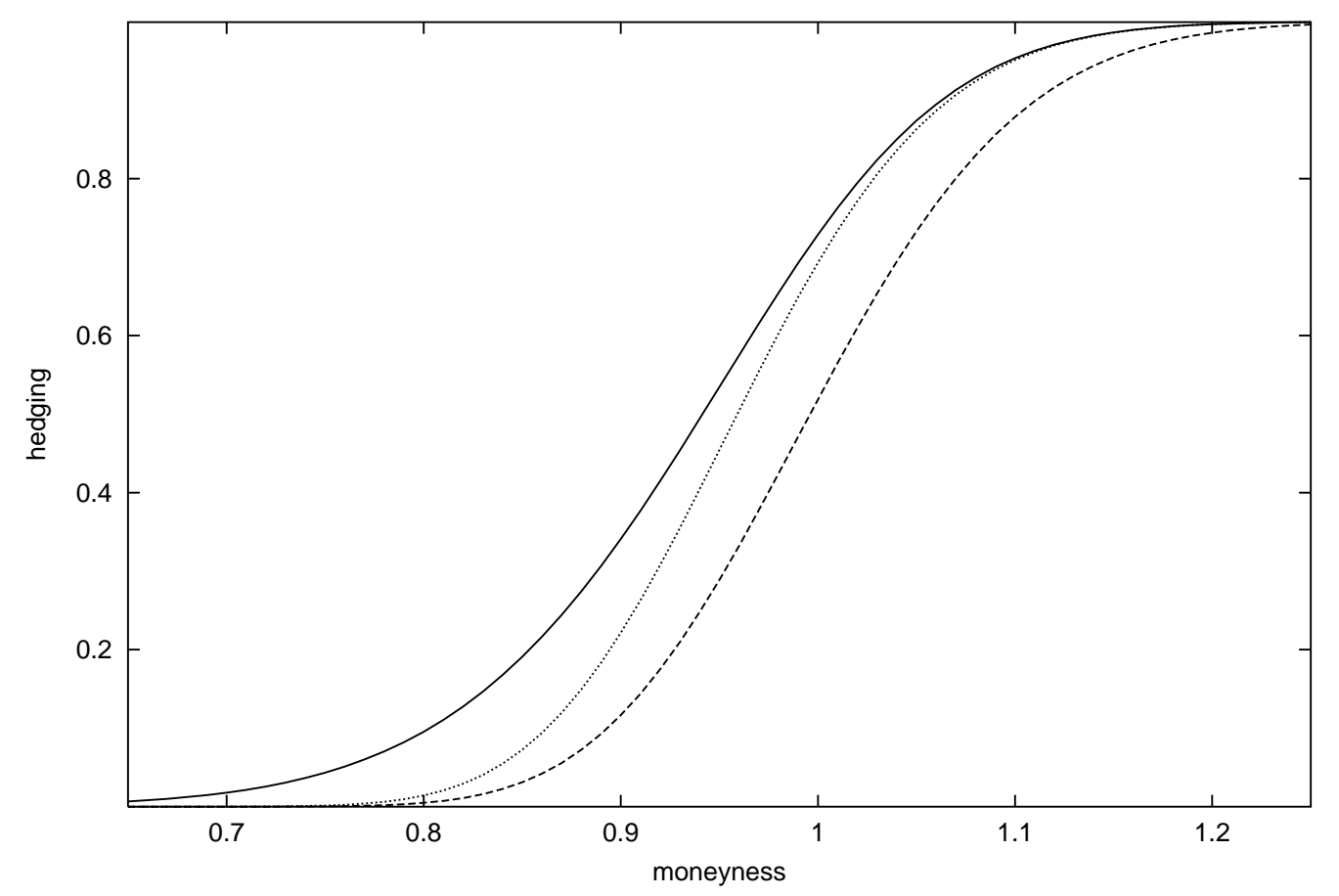

Figure 7. Hedging in terms of the moneyness. Psi hedging and delta hedging as a function of the moneyness $S / K$. The solid line represents psi hedging when $\tau=1$ day, the time to expiration is $T-t=20$ days, and the exercising price of the secondary call is $K^{\prime}=0.9 K$. The dotted line corresponds to the delta hedging still assuming the $\mathrm{O}-\mathrm{U}$ asset model with the same correlation and expiration time. The dashed line corresponds to B-S delta hedging ( $r$ and $\sigma$ as in Fig. 2).

is not expressed in terms of the final call price $C_{O U}=C(S, t)$. As we have shown in Section 5.3, psi hedging in terms of $C_{O U}$ can only be derived from the effective onedimensional process (23). In this case, the removal of the randomness coming from $d S$ implies that hedging is given by Eq. (55). Since $C_{S}=\delta_{O U}$, we see from Eqs. (55) and Eq. (65) that

$$
\psi(S, t)=\frac{N\left(d_{1}\right)}{N\left(d_{1}^{\prime}\right)} .
$$

Now, we take the secondary option to be an European call with maturity $T$ and exercising price $K^{\prime}<K$, where $T$ and $K$ refer to the primary option. We plot in Fig. 7 the psi hedging as a function of the moneyness. We see there that the $\psi$ hedging is always greater than $\delta_{O U}$ and $\delta_{B S}$ hedgings. Since $N\left(d_{1}^{\prime}\right) \rightarrow 1$ when $K^{\prime} \rightarrow 0$, the psi hedging approaches to the delta hedging $\delta_{O U}$ as the moneyness of the secondary call tends to infinity. This is consistent with the fact that secondary calls have the same price as the underlying stock when its exercising price is zero, i.e. $C^{\prime} \rightarrow S$ as $K^{\prime} \rightarrow 0$ (see Eq. (31)). Therefore, having secondary calls with exercising price equal to zero is equivalent to own underlying shares and the O-U psi hedging coincides with the $\mathrm{O}-\mathrm{U}$ delta hedging. 
As we have mentioned, psi hedging $\psi$ indicates the number of secondary calls per call to be hold if we follow a risk-free strategy with the modified portfolio (41). Therefore, the money invested to carry out this strategy is given by $\psi C^{\prime}$. That is

$$
\psi C^{\prime}=\frac{N\left(d_{1}\right)}{N\left(d_{1}^{\prime}\right)}\left[S N\left(d_{1}^{\prime}\right)-K^{\prime} e^{-r(T-t)} N\left(d_{2}^{\prime}\right)\right],
$$

where we have combined the Eqs. (31) and (69). On the other hand, delta hedging also indicates the number of shares per call to be hold in a risk-free strategy with the B-S portfolio (24). And, analogously, the money necessary to perform this strategy is

$$
\delta S=S N\left(d_{1}\right),
$$

where $\delta$ is given by Eq. (65). We compare these quantities in order to know which hedging is cheaper for the investor. From Eqs. (70) and (71), we see

$$
\frac{\psi C^{\prime}}{\delta S}=1-\frac{K^{\prime} e^{-r(T-t)} N\left(d_{2}^{\prime}\right)}{S N\left(d_{1}^{\prime}\right)},
$$

but

$$
0 \leq \frac{K^{\prime} e^{-r(T-t)} N\left(d_{2}^{\prime}\right)}{S N\left(d_{1}^{\prime}\right)} \leq 1 .
$$

Therefore, $\psi C^{\prime}<\delta S$ and psi hedging is always less expensive than delta hedging. Note that when $K^{\prime} \rightarrow 0$ both strategies have the same cost. In Fig. 8 we plot, as a function of moneyness, the relative psi hedging cost, $\psi C^{\prime} / K$, along with the relative delta hedging cost, $\delta S / K$. We see there that psi hedging is considerably less expensive than delta hedging and this difference increases with moneyness. Indeed, for an ATM call $(S / K=1.00)$ and with parameter values as that of Fig. 8, delta hedging is approximately $800 \%$ more expensive than psi hedging.

Combining Eqs. (34), (55) and (56) one can easily show that when $\tau=0$ the O-U psi hedging is $\psi_{B S}=N\left(d_{1}^{B S}\right) / N\left(d_{1}^{B S^{\prime}}\right)$ 四, where the prime refers to the secondary call. Since $\delta=C_{S}=N\left(d_{1}\right)$, we have

$$
\psi_{B S}=\frac{\delta_{B S}}{\delta_{B S}^{\prime}} .
$$

Finally, for the secondary call, whose exercising price goes to zero, $\delta_{B S}^{\prime} \rightarrow 1$ and, again, B-S psi hedging and B-S delta hedging coincide.

\section{Conclusions}

We have developed option pricing with perfect hedging in an inefficient market model. The inefficiency of the market is related to the fact that the underlying price variations are autocorrelated over an arbitrary time period $\tau$. In order to take these correlations into account we have modelled the underlying price $S(t)$ as a singular diffusion process

$\S$ This is straightforward to prove from Eq. (70) since $\psi C^{\prime} \geq 0$.

$\|$ We use the subscript $B S$ in $\psi_{B S}$ to indicate that this hedging refers to an uncorrelated stock $(\tau=0)$, as in the B-S world 


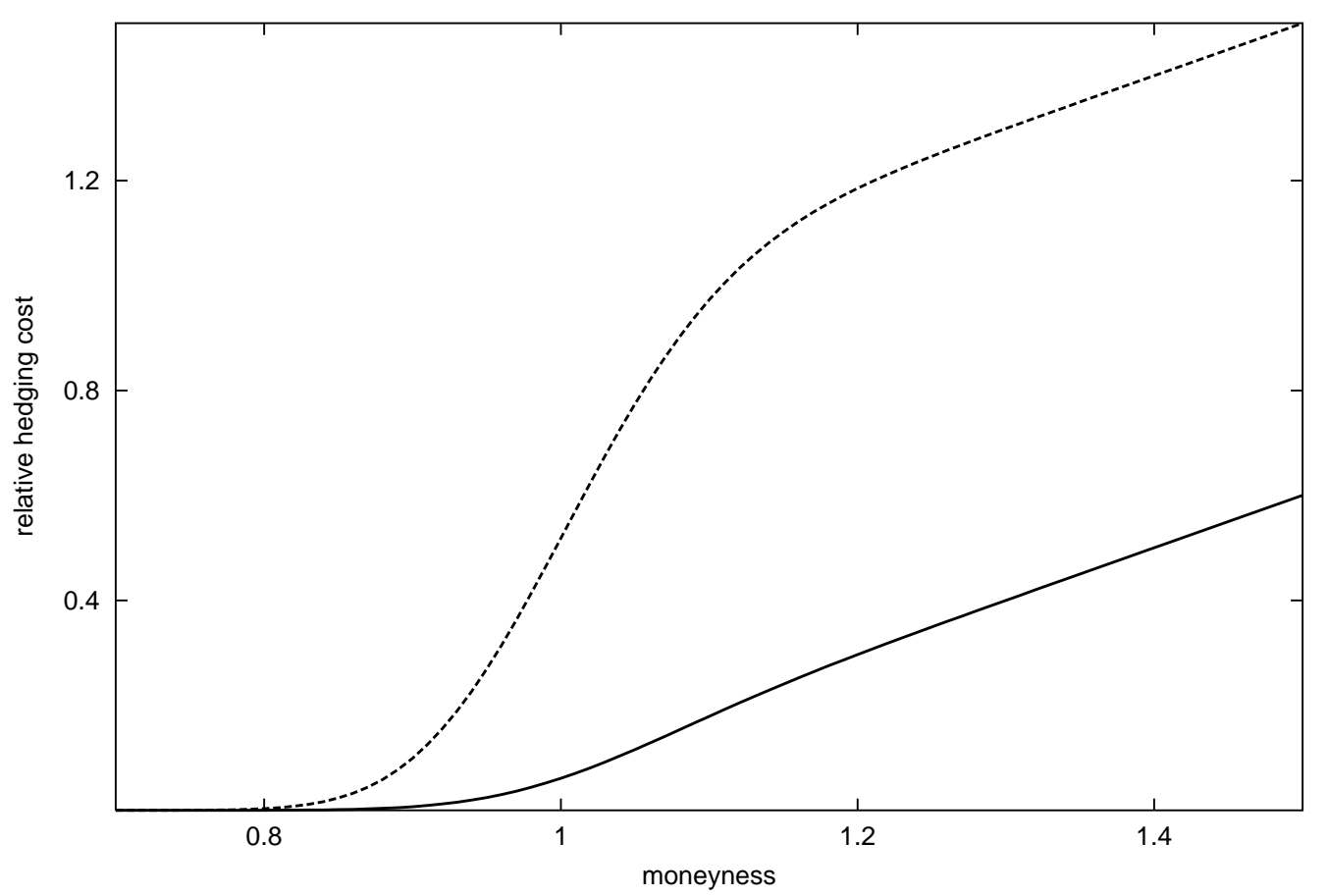

Figure 8. Relative hedging costs $\psi C^{\prime} / K$ and $\delta S / K$ as a function of the moneyness $S / K$. The solid line represent psi hedging cost when $\tau=1$ day, the time to expiration is $T-t=20$ days, and the exercising price of the secondary call is $K^{\prime}=0.9 K$. The dotted line corresponds to the delta hedging with $\tau=1$ day and $T-t=20$ days $(r$ and $\sigma$ as in Fig. 2).

in two dimensions (O-U process) instead of the standard assumption that $S(t)$ is a onedimensional diffusion given by the geometric Brownian motion with constant volatility.

The option pricing method has been developed by keeping perfect hedging with a riskless strategy which finally results in a closed and exact expression for the European call. Our pricing formula has the same functional form as the B-S price but replaces the variance of the Wiener process by the variance of the $\mathrm{O}-\mathrm{U}$ process. The $\mathrm{O}-\mathrm{U}$ variance, $\kappa(t)$, is smaller than the B-S variance, $\sigma^{2} t$, which implies that the equivalent volatility in the O-U case is lower than B-S volatility $\square$. But less volatility implies a lower option price. We have indeed proved that the $\mathrm{B}-\mathrm{S}$ call price is always greater than the $\mathrm{O}-\mathrm{U}$ price. In other words, the assumption of uncorrelated assets overprices the European call. This agrees with the fact that correlation, which can be regarded as a form of predictability, implies less risk and therefore a lower price for the option. We have quantified this overprice and showed that B-S formula notably overprices options and, more strikingly, that the overprice persists for a long time regardless of the strength of correlations. We have also analyzed the sensitivity of the O-U price to several conditions.

I Since the volatility $\sigma$ is the square root of the variance per unit time, one can define, in the O-U case, an equivalent volatility by $\sigma_{O U}=\sqrt{\dot{\kappa}(t)}$, where the dot denotes time derivative. From Eq. (11) we see that $\sigma_{O U} / \sigma=\sqrt{1-e^{-t / \tau}} \leq 1$. 
Thus we have proved that while $C_{O U}$ is more sensitive to changes in the interest rate and stock price than $C_{B S}$, it is also less sensitive to any change of the volatility. The practical consequences of this are nontrivial.

The option price and the hedging strategy have been obtained using two different approaches. The most straightforward way of getting the call price is by means of a projection onto a one-dimensional process with a time-varying volatility. A second way of obtaining the option price starts with the complete two-dimensional O-U process (2)(3). This is a longer procedure but opens the door to a new hedging strategy: the psi hedging. We have therefore two ways of acheiving the perfect hedging: the usual one consisting in holding underlying assets (delta hedging), and the second one which uses secondary calls instead of assets (psi hedging). We have shown that this last strategy can be considerable less expensive than the delta hedging and can avoid a possible lack of liquidity of underlying shares. Finally, the proportion of secondary calls to be held, i.e., the psi hedging, converges towards $\mathrm{O}-\mathrm{U}$ delta hedging when the exercising price of the secondary call tends to zero.

In practice our method of valuation requires the estimate of one more parameter, the correlation time, than in the B-S Wiener case. Assuming that the underlying asset is driven by $\mathrm{O}-\mathrm{U}$ noise one can find an estimate for the correlation time $\tau$ by evaluating the variance $\kappa(t)$ of the asset return. Once one has an estimate of this variance the correlation time is given in Eq. (11).

We finally mention that one interesting extension of the valuation method presented is to the American option. Although this case is more involved, one is probably able to obtain, at least an approximate or a numerical result using a combination of first passage times and martingale methods, as recently presented by Bunch and Johnson (2000). In any case we believe that the effects of autocorrelations on the valuation of an American option will be even more critical than for the European call. This case is under present investigation.

\section{Acknowledgments}

The authors acknowledge helpful comments and discussions with Alan McKane, Miquel Montero, Josep M. Porrà and Jaume Puig. We are particularly grateful to Santiago Carrillo and George H. Weiss for their many suggestions to improve the manuscript. This work has been supported in part by Dirección General de Proyectos de Investigación under contract No. BFM2000-0795, and by Generalitat de Catalunya under contract No. 2000 SGR-00023.

\footnotetext{
+ The construction of the portfolio with secondary calls is one simple way of proceeding. Obviously, any other secondary derivative on the same asset would serve.
} 


\section{Appendix A. Mathematical properties of the model}

We present some of the most important properties of the model given by the pair of stochastic equations in Eqs. (5) and (6). Their formal solutions are

$$
V(t)=V_{0} e^{-\left(t-t_{0}\right) / \tau}+\frac{\sigma}{\tau} \int_{t_{0}}^{t} e^{-\left(t-t^{\prime}\right) / \tau} d W\left(t^{\prime}\right),
$$

and

$$
\begin{aligned}
R(t)=\mu\left(t-t_{0}\right)+V_{0} \tau(1 & \left.-e^{-\left(t-t_{0}\right) / \tau}\right) \\
& +\frac{\sigma}{\tau} \int_{t_{0}}^{t} d t^{\prime} \int_{t_{0}}^{t^{\prime}} e^{-\left(t^{\prime}-t^{\prime \prime}\right) / \tau} d W\left(t^{\prime \prime}\right),
\end{aligned}
$$

where we have assumed that the process begun at time $t_{0}$ with initial velocity $V_{0}$ and return $R_{0}=0$. The return $R(t)$ has the following conditional mean value

$$
E\left[R(t) \mid V_{0}\right]=\mu\left(t-t_{0}\right)+\tau\left(1-e^{-\left(t-t_{0}\right) / \tau}\right) V_{0},
$$

and variance

$$
\operatorname{Var}\left[\mathrm{R}(\mathrm{t}) \mid \mathrm{V}_{0}\right]=\sigma^{2}\left[\left(\mathrm{t}-\mathrm{t}_{0}\right)-2 \tau\left(1-\mathrm{e}^{-\left(\mathrm{t}-\mathrm{t}_{0}\right) / \tau}\right)+\frac{\tau}{2}\left(1-\mathrm{e}^{-2\left(\mathrm{t}-\mathrm{t}_{0}\right) / \tau}\right)\right] .
$$

Since $(R(t), V(t))$ is a diffusion process in two dimensions, its joint density $p(R, V, t)$ satisfies the following Fokker-Planck equation (Gardiner (1985))

$$
p_{t}=-(\mu+V) p_{R}+\frac{V}{\tau} p_{V}+\frac{\sigma^{2}}{2 \tau^{2}} p_{V V}
$$

This is to be solved subject to the initial conditions $R\left(t_{0}\right)=0$ and $V\left(t_{0}\right)=V_{0}$, that is

$$
p\left(R, V, t_{0} \mid V_{0}, t_{0}\right)=\delta(R) \delta\left(V-V_{0}\right) .
$$

A first step towards solving the problem (A.2)-(A.3) is the definition of the joint Fourier transform

$$
\tilde{p}(\alpha, \beta, t)=\int_{-\infty}^{\infty} d R e^{i \alpha R} \int_{-\infty}^{\infty} d V e^{i \beta V} p(R, V, t) .
$$

Then problem (A.2)-(A.3) becomes

$$
\begin{aligned}
& \partial_{t} \tilde{p}=i \alpha \mu \tilde{p}+(\alpha-\beta / \tau) \alpha \partial_{\beta} \tilde{p}-\left(\sigma^{2} / 2 \tau^{2}\right) \beta^{2} \tilde{p}, \\
& \tilde{p}(\alpha, \beta, t=0)=e^{i \beta V_{0}} .
\end{aligned}
$$

We look for a solution of the form

$$
\begin{aligned}
\tilde{p}(\alpha, \beta, t)= & \exp \left\{i\left[\alpha m_{1}(t)+\beta m_{2}(t)\right]\right\} \\
& \times \exp \left\{-\left[K_{11}(t) \alpha^{2}+K_{12}(t) \alpha \beta+K_{22}(t) \beta^{2}\right] / 2\right\},
\end{aligned}
$$

where $m_{i}(t)$ and $K_{i j}(t)$ are functions to be determined. We substitute Eq. A.6) into (A.4) and identify term by term. We have

$$
\begin{array}{ll}
\dot{m}_{1}=\mu+m_{2}, & \dot{m}_{2}=-m_{2} / \tau ; \\
\dot{K}_{22}+(2 / \tau) K_{22}=\sigma^{2} \tau^{2}, & \dot{K}_{12}+(1 / \tau) K_{12}=2 K_{22}(t), \quad \dot{K}_{11}=2 K_{12},
\end{array}
$$


with the intial conditions, according to Eqs. (A.5)-(A.6), given by

$$
m_{2}(0)=V_{0}, \quad m_{1}(0)=K_{i j}(0)=0 \quad(i, j=1,2) .
$$

The solution reads

$$
m_{1}(t)=\mu t+V_{0} \tau\left(1-e^{-t / \tau}\right), \quad m_{2}(t)=V_{0} e^{-t / \tau},
$$

and $K_{i j}(t)$ are given by

$$
\begin{aligned}
& K_{11}(t)=\sigma^{2}\left[t-2 \tau\left(1-e^{-t / \tau}\right)+\frac{\tau}{2}\left(1-e^{-2 t / \tau}\right)\right], \\
& K_{12}(t)=\frac{\sigma^{2}}{2}\left(1-e^{-t / \tau}\right)^{2}, \quad K_{22}(t)=\frac{\sigma^{2}}{2 \tau}\left(1-e^{-2 t / \tau}\right),
\end{aligned}
$$

The inverse Fourier transform of Eq. (A.6) yields the Gaussian density

$$
\begin{gathered}
p\left(R, V, t \mid V_{0}, t_{0}\right)=\frac{1}{\left.2 \pi \sqrt{\operatorname{det}\left[\boldsymbol{K}\left(t-t_{0}\right)\right.}\right]} \exp \left\{-\frac{\left(V-V_{0} e^{-\left(t-t_{0}\right) / \tau}\right)^{2}}{2 K_{22}\left(t-t_{0}\right)}\right. \\
\left.-\frac{\left[K_{22}\left(t-t_{0}\right)\left(R-m\left(t-t_{0}, V_{0}\right)\right)-K_{11}\left(t-t_{0}\right)\left(V-V_{0} e^{-\left(t-t_{0}\right) / \tau}\right)\right]^{2}}{2 K_{22}\left(t-t_{0}\right) \operatorname{det}\left[\boldsymbol{K}\left(t-t_{0}\right)\right]}\right\},
\end{gathered}
$$

where

$$
\operatorname{det}[\boldsymbol{K}(t)] \equiv K_{11}(t) K_{22}(t)-K_{12}^{2}(t)
$$

and

$$
m\left(t, V_{0}\right)=\mu t+V_{0} \tau\left(1-e^{-t / \tau}\right) .
$$

Notice that the joint density (A.9) is a function of the time differences $t-t_{0}$ where $t_{0}$ is the initial observation time, so that the two-dimensional diffusion $(S(t), V(t))$ is a time homogeneous process and, without loss of generality, we may assume that $t_{0}=0$.

The marginal pdf of the velocity $V(t)$,

$$
p\left(V, t \mid V_{0}\right)=\int_{-\infty}^{\infty} p\left(R, V, t \mid V_{0}\right) d R
$$

is

$$
p\left(V, t \mid V_{0}\right)=\frac{1}{\sqrt{2 \pi K_{22}(t)}} \exp \left[-\frac{\left(V-V_{0} e^{-t / \tau}\right)^{2}}{2 K_{22}(t)}\right] .
$$

In the stationary regime $(t \rightarrow \infty)$ we find a normal density independent of the initial velocity:

$$
p_{s t}(V)=\frac{1}{\sqrt{\pi\left(\sigma^{2} / \tau\right)}} e^{-\tau V^{2} / \sigma^{2}} .
$$

Analogously, the marginal density of the return $R(t)$,

$$
p\left(R, t \mid V_{0}\right)=\int_{-\infty}^{\infty} p\left(R, V, t \mid V_{0}\right) d V
$$


is

$$
p\left(R, t \mid V_{0}\right)=\frac{1}{\sqrt{2 \pi K_{11}(t)}} \exp \left\{-\frac{\left[R-m\left(t, V_{0}\right)\right]^{2}}{2 K_{11}(t)}\right\} .
$$

If we assume that the initial velocity $V_{0}=V(0)$ is a random variable distributed according to the pdf in Eq. (A.13). We can therefore average the above densities to obtain a pdf independent of $V_{0}$. That is,

$$
p(R, V, t)=\int_{-\infty}^{\infty} p\left(R, V, t \mid V_{0}\right) p_{s t}\left(V_{0}\right) d V_{0}
$$

and similarly for the marginal pdf's $p(R, t)$ and $p(V, t)$. Since we are mainly interested on the marginal distribution of the return we will give its explicit expression. Thus, from Eqs. (A.13) and (A.14) we have

$$
p(R, t)=\frac{1}{\sqrt{2 \pi \kappa(t)}} \exp \left[-\frac{(R-\mu t)^{2}}{2 \kappa(t)}\right],
$$

where $\kappa(t)$ is given by Eq. (11). Alternatively, the distribution of the underlying price $S=S_{0} e^{R}$ is given by the log-normal density

$$
p\left(S, t \mid S_{0}\right)=\frac{1}{S \sqrt{2 \pi \kappa(t)}} \exp \left[-\frac{\left(\ln S / S_{0}-\mu t\right)^{2}}{2 \kappa(t)}\right] .
$$

From this we easily see that the conditional probability $p\left(S^{\prime}, T \mid S, t\right)$ when $t \leq T$ is

$$
p\left(S^{\prime}, T \mid S, t\right)=\frac{1}{S^{\prime} \sqrt{2 \pi \kappa(T-t)}} \exp \left[-\frac{\left[\ln S^{\prime} / S-\mu(T-t)\right]^{2}}{2 \kappa(T-t)}\right] .
$$

\section{Appendix B. The Itô formula for processes driven by O-U noise}

In this Appendix we generalize the Itô formula for processes driven by OrnsteinUhlenbeck noise. This is applied to the share price $S(t)$ which is governed by the pair of stochastic equations (2)-(3)

$$
d S(t)=S(\mu+V) d t, \quad d V(t)=-\frac{V}{\tau} d t+\frac{\sigma}{\tau} d W .
$$

Consider a generic function $f(S, V, t)$ which depends on all of the variables that characterize the underlying asset. The differential of $f(S, V, t)$ is defined by

$$
d f(S, V, t) \equiv f(S(t+d t), V(t+d t), t+d t)-f(S(t), V(t), t) .
$$

But the Taylor expansion of (B.2) yields

$$
\begin{aligned}
d f(S, V, t)=f_{S} d S+ & f_{V} d V+f_{t} d t \\
& +\frac{1}{2} f_{S S} d S^{2}+\frac{1}{2} f_{V V} d V^{2}+f_{S V} d S d V+\cdots,
\end{aligned}
$$

where the expansion also involves higher order differentials such as $(d t)^{2},(d S)^{3},(d V)^{3}$, etc. However, the differential of the Wiener process, $d W$, satisfies the well-known property, in the mean-square sense, $d W(t)^{2}=d t$ (Gardiner (1985)). And from the 
pair of equations (B.1) we then see that $d S^{2}$ is of order $d t^{2}$ while $d V^{2}$ is of order $d t$ and $d S d V$ is of order $d t^{3 / 2}$. Therefore, up to order $d t$, Eq. (B.3) reads

$$
d f(S, V, t)=f_{S} d S+f_{V} d V+f_{t} d t+\frac{\sigma^{2}}{2 \tau^{2}} f_{V V} d t,
$$

which is the Itô formula for our singular two-dimensional process (2)-(3).

Suppose now we start from the effective one-dimensional SDE (17)

$$
d R(t)=\mu d t+\sqrt{\dot{\kappa}(T-t)} d W(t) .
$$

We will prove that the corresponding $\mathrm{SDE}$ for the stock price defined as $S=S_{0} e^{R}$ is given by Eq. (23). In effect, substituting Eq. (B.5) in the Taylor expansion

$$
d S(R)=S_{R} d R+\frac{1}{2} S_{R R} d R^{2}+\cdots,
$$

neglecting orders higher than $d t$ and taking into account that $d R^{2}=\dot{\kappa}(T-t) d t$ (in mean square sense), we finally obtain

$$
\frac{d S(t)}{S(t)}=[\mu+\dot{\kappa}(T-t) / 2] d t+\sqrt{\dot{\kappa}(T-t)} d W(t),
$$

which is Eq. (23).

Moreover, we can also give the differential of a generic function $f(S, t)$ when underlying obeys SDE (B.6). In this case, we have

$$
d f(S, t)=f_{S} d S+f_{t} d t+\frac{1}{2} \dot{\kappa}(T-t) S^{2} f_{S S} d t,
$$

where again we have neglected higher order contributions than $d t$.

\section{Appendix C. A derivation of the risk premium}

We proceed to find a closed expression for the arbitrary function $\lambda(S, V, t)$ that appears in Eq. (47). The call price $C$ is a function of $S, V$, and $t$. We now consider this function taking into account that $S=S(t)$ and $V=V(t)$ follow Eqs. (2) and (3), respectively. This therefore allows us to evaluate the random differential $d C$ using the Itô lemma, as a result we find that

$$
d C=\left[C_{t}+(\mu+V) S C_{S}+\frac{\sigma^{2}}{2 \tau} C_{V V}\right] d t+C_{V} d V .
$$

After using Eqs. (47) and (3), we have

$$
d C=\left[r C+\left(\lambda-\frac{V}{\tau}\right) C_{V}\right] d t+\frac{\sigma}{\tau} C_{V} d W .
$$

The expected value of $d C$, on the assumption that $C(t)=C$ is known, reads

$$
E[d C \mid C]=\left[r C+\left(\lambda-\frac{V}{\tau}\right) C_{V}\right] d t .
$$

We claim that this average must grow at the same rate as the risk-free bond:

$$
E[d C \mid C]=r C d t,
$$


since otherwise the option would not be in equilibrium (Hull (2000)). In some sense, this assumption is similar to that of the equivalent martingale measure demand expecting that markets grow in average as the risk-free bond (Harrison and Pliska (1981)).

The substitution of Eq. (C.3) into Eq. (C.2) yields the following expression for the risk premium $\lambda(S, V, t)$ :

$$
\lambda=\frac{V}{\tau} \text {. }
$$

\section{Appendix D. Solution to the problem in Eqs. (49)-(30)}

We will solve Eq. (49) subject to the final condition in Eq. (30). Define a new independent variable $Z$

$$
S=e^{Z}
$$

where the domain of $Z$ is unrestricted. The problem posed in Eqs. (49)-(30) now reads

$$
\begin{aligned}
& C_{t}=r C-(\mu+V) C_{Z}+\frac{V}{\tau} C_{V}-\frac{\sigma^{2}}{2 \tau} C_{V V}, \\
& C(Z, V, T)=\max \left[e^{Z}-K, 0\right] .
\end{aligned}
$$

The solution to this problem can be written in the form

$$
C(Z, V, t)=\int_{-\infty}^{\infty} d Z^{\prime} \int_{-\infty}^{\infty} d V^{\prime} \max \left[e^{Z^{\prime}}-K\right] G\left(Z, V, t \mid Z^{\prime}, V^{\prime}, T\right),
$$

where $G\left(Z, V, t \mid Z^{\prime}, V^{\prime}, T\right)$ is the Green function for the problem (Mynt-U (1987)), i.e., $G\left(Z, V, t \mid Z^{\prime}, V^{\prime}, T\right)$ is the solution to

$$
G_{t}=r G-(\mu+V) G_{Z}+\frac{V}{\tau} G_{V}-\frac{\sigma^{2}}{2 \tau} G_{V V}
$$

with the final condition

$$
G\left(Z, V, T \mid Z^{\prime}, V^{\prime}, T\right)=\delta\left(Z-Z^{\prime}\right) \delta\left(V-V^{\prime}\right),
$$

where $\delta\left(X-X^{\prime}\right)$ is the Dirac delta function. Define $\bar{G}=e^{-r t} G$, then the final-value problem in Eqs. (D.5) and (D.6) reads

$$
\begin{aligned}
& \bar{G}_{t}=-(\mu+V) \bar{G}_{Z}+\frac{V}{\tau} \bar{G}_{V}-\frac{\sigma^{2}}{2 \tau} \bar{G}_{V V}, \\
& \bar{G}\left(Z, V, T \mid Z^{\prime}, V^{\prime}, T\right)=e^{-r T} \delta\left(Z-Z^{\prime}\right) \delta\left(V-V^{\prime}\right) .
\end{aligned}
$$

Note that Eq. (D.7) is the backward equation corresponding to Eq. (A.2). Therefore, Eq. (A.9) permits us to write the solution to the problem posed in Eqs. (D.7)-(D.8) (Gardiner (1985)). This solution implies that $G$ is

$$
\begin{gathered}
G\left(Z, V, t \mid Z^{\prime}, V^{\prime}, T\right)=\frac{1}{2 \pi \sqrt{\operatorname{det}[\boldsymbol{K}(T-t)}]} \exp \left\{-r(T-t)-\frac{\left[V^{\prime}-V e^{-(T-t) / \tau}\right]^{2}}{2 K_{22}(T-t)}\right. \\
\left.-\frac{\left[K_{22}(T-t)\left(Z^{\prime}-Z+m(T-t, V)\right)-K_{11}(T-t)\left(V^{\prime}-V e^{-(T-t) / \tau}\right)\right]^{2}}{2 K_{22}(T-t) \operatorname{det}[\boldsymbol{K}(T-t)]}\right\},
\end{gathered}
$$


where $\operatorname{det}[\boldsymbol{K}(t)], K_{i j}(t)$, and $m(t, V)$ are defined in Eqs. (A.10)-(A.11).

Substituting Eq. (D.9) into Eq. (D.4) and finally reverting to the original variables we obtain Eq. (50) with

$$
z_{1}=\frac{\ln (S / K)+m(T-t, V)+K_{11}(T-t)}{\sqrt{K_{11}(T-t)}}, \quad z_{2}=z_{1}-\sqrt{K_{11}(T-t)} .
$$

Finally it can be shown, after some lengthy but simple manipulations, that the functions $\bar{z}_{1,2}=\bar{z}_{1,2}(S, T-t)$ appearing in the averaged price $\bar{C}(S, t)$, Eq. (52), are given by

$$
\bar{z}_{1}=\frac{\ln (S / K)+\mu(T-t)+\kappa(T-t)}{\sqrt{\kappa(T-t)}}, \quad \bar{z}_{2}=\bar{z}_{1}-\sqrt{\kappa(T-t)},
$$

where $\kappa(t)$ is given in Eq. (11).

\section{References}

Aurell, E., R. Baviera, O. Hammarlid, M. Serva, and A. Vulpiani, 2000, A General Methodology to Price and Hedge Derivatives in Incomplete Markets, International Journal of Theoretical and Applied Finance 3, 1-24.

Black, F., and M. Scholes, 1973, The Pricing of Options and Corporate Liabilities, Journal of Political Economy 81, 637-659.

Björk, T., 1998, Arbitrage Theory in Continuous Time (Oxford University Press, Oxford, UK).

Bouchaud, J. P., and M. Potters, 2000, Theory of Financial Risks (Cambridge University Press, Cambridge, UK).

Breen, W., and R. Jagannathan, 1989, Economics significance of predictable variations in stock index returns, Journal of Finance 44, 1177-1189.

Bunch, D. S., and H. Johnson, 2000, The American Put Option and Its Critical Stock Price, Journal of Finance 55, 2333-2356.

Campbell, J., and Y. Hamao, 1992, Predictable stock returns in the United States and Japan: A study of long-term capital market integration, Journal of Finance 47, 43-70.

Cootner, P. H., 1964, The Random Character of Stock Market Prices, (M.I.T. Press, Cambridge, MA).

Cox, J. C., and S. A. Ross, 1976, The Valuation of Options for Alternative Stochastic Processes, Journal of Financial Economics 3, 145-166.

Doob, J. L., 1942, The Brownian Movement and Stochastic Equations, Annals of Mathematics 43, 351-369.

Dumas, B., J. Fleming, and R. Whaley, 1998, Implied Volatility Smiles: Some Empirical Tests, Journal of Finance 53, 2059-2106.

Fama, E. F., 1963, Mandelbrot and The Stable Paretian Hypothesis, Journal of Business 36, 420-429.

— 1965, The Behaviour of Stock Market Prices, Journal of Business 38, 34-105.

—_, 1991, Efficient Capital Markets II, Journal of Finance 46, 1575-1617.

Feller, W., 1954, Diffusion processes in one dimension, Transactions of the American Mathematical Society 77, 1-31.

Figlewski, S., 1989, Options Arbitrage in Imperfect Markets, Journal of Finance 64, 1289-1311.

Gardiner, C. W., 1985, Handbook of Stochastic Methods (Springer-Verlag, Heilderberg, Berlin, New York).

Ghysels, E., A. C. Harvey, and E. Renault, 1996, Stochastic volatility, in G. S. Mandala and C. R. Rao eds: Statistical Methods in Finance (North-Holland, Amsterdam, New York).

Grossman, S. J., and J. E. Stiglitz, 1980, On the Impossibility of Informationally Efficient Markets, The American Economic Review 70, 222-227. 
Harrison, J. M., and D. Kreps, 1979, Martingales and Multiperiod Securities Markets, Journal of Economic Theory 20, 381-408.

Harrison, J. M., and S. R. Pliska, 1981, Martingales and Stochastic Integrals in the Theory of Continuous Trading, Stochastic Processes and Their Applications 11, 215-260.

Harrison, J. M., R. Pitbladdo and S. M. Schaefer, 1984, Continuous Price Processes in Frictionless Markets Have Infinite Variation, Journal of Business 57, 353-365.

Heston, S., 1993, A Closed-Form Solution for Options with Stochastic Volatility to Bond and Currency Options, Review of Financial Studies 6, 327-343.

Heston, S., and S. Nandi, 2000, A Closed-Form GARCH Option Valuation Model, Review of Financial Studies 13, 585-625.

Hull, J. C., and A. White, 1987, The Pricing of Options with Stochastic Volatilities, Journal of Finance 42, 281-300.

Hull, J. C., 2000, Options, Futures, \& Other Derivatives (Prentice-Hall, Upper Saddle River, NJ).

Lighthill, M. J., 1958, An Introduction to Fourier Analysis and Generalized functions (Cambridge University Press, Cambridge, UK).

Lo, A. W., and J. Wang, 1995, Implementing Option Pricing Models When Asset Returns Are Predictable, Journal of Finance 50, 87-129.

Mandelbrot, B., 1963, The Variation of Certain Speculative Prices, Journal of Business 36, 394-419.

Markowitz, H., 1952, Portfolio Selection, Journal of Finance 7, 77-91.

Merton, R. C., 1973a, Theory of Rational Option Pricing, Bell Journal of Economics 4, 141-183.

— 1973b, Appendix: Continuous-Time Speculative Processes, SIAM Review 1, 34-38.

— 1976, Option Pricing when Underlying Stock Returns are Discontinuous, Journal of Financial Economics 3, 125-144.

Mynt-U, Tyn, 1987, Partial Differential Equations for Scientists and Engineers (North-Holland, Amsterdam, New York).

Ross, S., 1976, Arbitrage Theory of Capital Asset Pricing, Journal of Economic Theory 13, 341-360.

Sharpe, W. F., 1964, Capital Asset Prices, Journal of Finance 19, 425-442.

Scott, L.O., 1987, Option Pricing when the Variance Changes Randomly: Theory, Estimation, and Application, Journal of Financial and Quantitative Analysis 22, 419-438.

Stein, E. M., and J. C. Stein, 1991, Stock Price Distributions with Stochastic Volatility: An Analytic Approach, Review of Financial Studies 4, 727-752.

Stratonovich, R. L., 1963, Topics in the Theory of Random Noise (Gordon and Breach, New York).

Uhlenbeck, G. E., and L. S. Ornstein, 1930, On the Theory of the Brownian Motion, Physical Review 31, 823-841. 